\title{
A New Hybrid Model Predictive Controller Design for Adaptive Cruise of Autonomous Electric Vehicles
}

\author{
Yuanhang Chen $(\mathbb{D}$, Guodong Feng, Shaofang $W u(\mathbb{D}$, and Xiaojun Tan \\ School of Intelligent Systems Engineering, Sun Yat-sen University, Guangzhou 510006, China \\ Correspondence should be addressed to Shaofang Wu; wushf7@mail.sysu.edu.cn
}

Received 20 October 2020; Revised 28 January 2021; Accepted 23 February 2021; Published 15 March 2021

Academic Editor: Bin Yu

Copyright (c) 2021 Yuanhang Chen et al. This is an open access article distributed under the Creative Commons Attribution License, which permits unrestricted use, distribution, and reproduction in any medium, provided the original work is properly cited.

\begin{abstract}
Autonomous driving is an appealing research topic for integrating advanced intelligent algorithms to transform automotive industries and human commuting. This paper focuses on a hybrid model predictive controller (MPC) design for an adaptive cruise. The driving modes are divided into following and cruising, and the MPC algorithm based on simplified dual neural network (SDNN) and proportional-integral-derivative (PID) based on single neuron (SN) are applied to the following mode and the cruising mode, respectively. SDNN is used to accelerate the solution of the quadratic programming (QP) problem of the proposed MPC algorithm to improve the computation efficiency, while PID based on SN performs well in the nonlinear and timevarying conditions in the ACC system. Moreover, lateral dynamics control is integrated into the designed system to fulfill cruise control in the curved road conditions. Furthermore, to improve the energy efficiency of the electric vehicle, an energy feedback strategy is proposed. The simulation results show that the proposed ACC system is effective on both straight roads and curved roads.
\end{abstract}

\section{Introduction}

Nowadays, to enhance traffic efficiency, improve driving comfort and energy economy, and ensure driving safety, various advanced automotive electronic control systems have been applied to vehicles on sale. For example, antilock brake system (ABS) and electronic stability program (ESP) have been widely used in passenger vehicles which gradually become standard configuration [1]. In this context, the concept of an advanced driving assistance system (ADAS) is proposed and gradually becomes a hot topic. As a significant component of ADAS, adaptive cruise control (ACC) owns the ability to execute acceleration and brake operations automatically, which can reduce driver's workloads, prevent traffic accidents, and increase economy efficiency.

ACC systems were widely investigated in the literature. Fancher [2] divided driving conditions of the ACC system into six types. In view of the differences of control modes at various working conditions, the author sets different switching rules in different working conditions based on the real vehicle experiments. Behnam [3] proposed to improve the performance of the ACC system by adopting the sliding mode controller. Swarm optimization theory was used to define the self-tuning parameters of the controller, and the sliding mode control was applied to the design of the feedback controller. In the sliding mode control, the sliding variable converged to zero, then the designed ACC controller was applied to the nonlinear vehicle model. The simulation results showed that the proposed control method worked well. Melanes [4] proposed the cooperative adaptive cruise system (CACC). By adding vehicle-mounted wireless communication to provide additional information to expand the range of sensor data and yield better performance, the approach could not only help to reduce traffic accidents, but also improve traffic flow. Bichi [5] proposed the stochastic model predictive control (SMPC). The serial hybrid electric vehicle was taken as the research object and the deterministic dynamic power system model was established. The driver behavior was seen as a dynamic random system that influenced the performance of the vehicle, and the 
driver behavior model was established to predict the dynamics of the leading vehicle and the power of future request, which improved the performance of powertrain control algorithm.

To achieve the prediction of the expected speed and the expected distance, based on the theory of the nonlinear model predictive control (NMPC), Shakouri [6] built the NMPC equation on a linear model corresponding to the operation mode, and ACC switched to cruise control (CC) smoothly. In order to verify the actual effect, specific tests were set up according to different driving scenarios, and, finally, the proposed system was proved to have good adaptability and reliability. Martinez [7] proposed a longitudinal motion control method of an automobile based on the reference model. An external reference model was designed independently for collaborative control of comfort and safety, and a closed-loop control system was designed independently for matching the actual system with the external reference model. Although this method provided consideration to both comfort and safety, the accuracy of the reference model was not satisfactory; the control system was complex; and the real-time control could not be guaranteed. Kim [8] proposed a method of virtual information of the following vehicle and the leading vehicle. Furthermore, he used the linear quadratic algorithm with the weight-varying coefficient to enable the following vehicle to track the leading vehicle smoothly, which had obvious effects on improving the comfort and fuel economy of the ACC system. Clement [9] proposed to design the optimal controller based on the multiobjective Pareto optimization strategy to solve the contradiction between economy and speed. Kim [10] built a driver model and used the real driver data to identify the model parameters through a self-learning algorithm while automatically adjusting the parameters of the controller.

On the basis of the second-order kinematics model, adopting the relative speed, the relative distance, and the relative acceleration between the following vehicle and the leading vehicle, Li derived the third-order longitudinal motion model and the performance function in [11]. Compared with the secondorder model, the control precision of the third-order model was higher in the control of the relative distance between vehicles. Zhao [12] built an optimized neural network model and trained it on the basis of supervised adaptive dynamic programming (SADP) algorithm. The result showed the proposed ACC system could limit the human behavior closely. Hu [13] constructed the vehicle-following model based on the Markov process and designed a supervised reinforcement learning controller based on the shaping method to realize adaptive cruise control considering the driving habits of different drivers. And the system showed a good control effect in the scene simulation test of following the leading vehicle, starting and stopping, and changing the driver.

Pointing at the ACC system in the low-speed range, Milanes [14] presented a comparative study between robust control method and other methods including classic PI control, a new control method called i-PI, fuzzy control, and neural fuzzy control based on expert knowledge to solve the problem of automatic operation of the accelerator pedal and brake pedal under low-speed condition. Kural [15] proposed the ACC system prediction model based on a multiobjective control strategy and applied the hierarchical control structure to conduct real-time optimization considering the minimum tracking error for vehicle control. Kanjee [16] proposed a method to divide the control of cruising and braking into three different parts, namely, cruise control, adaptive cruise control, and braking control in cruising on the basis of the time headway. And in the research model, the operating characteristics of the individual driver could be defaulted, respectively, according to different drivers which made cruise control safer. Vajedi and Azad [17] applied the NMPC technology to the speed control of the ACC system and adopted Pontryagin minimum theory optimization to improve traffic safety and fuel economy.

In addition, advanced techniques such as light autobody lightweight design have been applied to reduce the fuel consumption over the past few decades for fuel vehicles, which were advantageous for ACC strategies in fuel vehicles [18-23]. Generally, most available ACC systems are based on fuel vehicles. However, intelligent vehicles can be adapted from electric vehicles (EV) easily as they tend to have better control characteristics and higher economy efficiency. Therefore, it is necessary to do more research on the ACC designed for full-electric vehicles.

Conventional ACC systems based on proportional-integralderivative (PID) are widely used in practice. However, they cannot meet the demand of incorporating the performances of safety, comfort, tracking, and energy economy. Though lots of researchers have studied energy management of fuel vehicles and hybrid vehicles [24], a few conventional ACC systems take battery energy management into consideration in the design of control strategies. In this paper, a neural-network based hybrid MPC is proposed for the adaptive cruise of autonomous electric vehicles. Simplified dual neural network (SDNN) technique is adopted to improve the following control, and PID based on single neuron ( $\mathrm{SN}$ ) is used to optimize cruising process. The proposed control takes the above-mentioned four key factors into a unified performance optimization framework and further formulates the optimization procedure into a quadratic programming $(\mathrm{QP})$ solution with spatiotemporal constraints in the following process.

Considering the high real-time requirements for the proposed ACC system in actual application, the computation complexity of the conventional MPC is unsatisfactory due to its rolling optimization. SDNN owns the advantage of occupying fewer resources and the algorithm is convenient to implement, so the SDNN algorithm is used to accelerate the computation of the quadratic programming routine of the Incremental MPC computation, which is helpful for vehicular embedded systems. Moreover, the vehicle dynamics is nonlinear and time-varying, conventional PID controllers cannot achieve the desired control effect in the cruising mode. Theoretically, single neuron can approach any nonlinear function infinitely, which ensures robustness and better performance while cruising, so the PID controller based on SN is designed for the cruising mode in this paper. Meanwhile, regenerative braking is taken into consideration by presenting an energy feedback strategy. Lateral dynamics control is also considered, which is often ignored by 
conventional ACC systems, so the proposed ACC system can work on the curved road.

The remaining parts of this paper are organized as follows: Section 2 presents the vehicle dynamical model and the braking strategy. Section 3 focuses on the Incremental MPC controller based on SDNN in the following mode, the PID controller based on SN in the cruising mode, and lateral dynamics control. Illustrative examples are given in Section 4. Finally, Section 5 concludes this paper.

\section{Model for Vehicle Longitudinal Dynamics and Braking Strategy}

2.1. Model for Vehicle Longitudinal Dynamics. This paper considers a passenger EV equipped with the ACC system. For the sake of the controller design, assume that (1) there is no lateral movement of the vehicle; (2) the vehicle runs on a straight road and the ground adhesion is sufficient to satisfy the movement of the vehicle; (3) the influence of tire inertia and tire rolling resistance moment is ignored; and (4) vehicle road slope angle is $\alpha$.

The forces exerted on the vehicle while driving is presented in Figure 1. Parameters in the vehicle longitudinal dynamics and braking strategy are given in Table 1 .

The vehicle longitudinal dynamic model can be expressed as

$$
m a_{\exp }=F_{t}-\frac{1}{21.15} C_{D} A v^{2}-m g f \cos \alpha-m g \sin \alpha-\delta m \frac{\mathrm{d} v}{\mathrm{~d} t} .
$$

According to (1), the desired driving torque based on the simplified vehicle longitudinal dynamic model is

$$
T_{t}^{d}=\frac{R}{i_{t} \eta}\left[\frac{1}{21.15} C_{D} A v^{2}+m g(f \cos \alpha+\sin \alpha)+m \delta a_{\exp }\right] .
$$

In the situation of braking, the desired braking torque is

$$
T_{b}^{d}=\frac{R}{i_{t} \eta}\left|-m a_{\exp }-\frac{1}{21.15} C_{D} A v^{2}-m g f\right| .
$$

The ground deceleration of the following vehicle in a range of normal driving speed is shown in Figure 2. $a_{\exp }$ is the expected acceleration of the following vehicle; $a\left(v_{1}\right)$ is the natural deceleration of the following vehicle at $v_{1}$. If $a_{\exp }>a\left(v_{1}\right)$, the following vehicle accelerates, else it decelerates.

For the electric vehicle model, its powertrain consists of a permanent magnet synchronous motor (PMSM), fixed ratio transmission, differential mechanism, and so on. Moreover, the vehicle is driven by the front wheel. The vehicle powertrain system is presented in Figure 3.

2.2. Braking Strategy. There are three kinds of electric vehicle braking in the ACC system: mechanical braking, regenerative braking, and hybrid braking. The ideal braking process of an electric vehicle is the linear change of braking force and the relation between the expected braking force and the braking strength is as

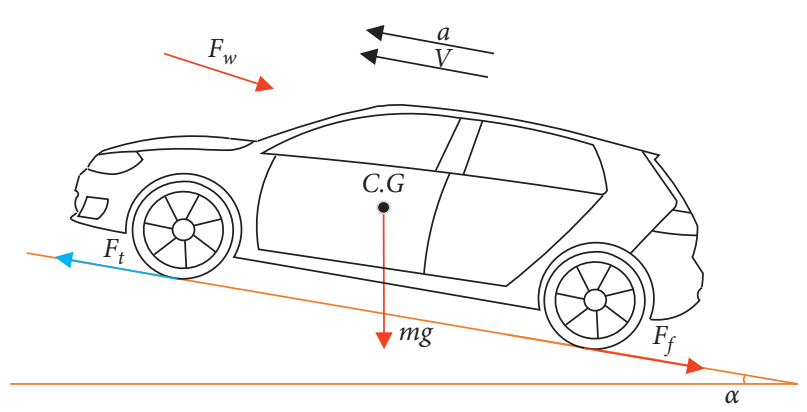

FIGURE 1: Schematic diagram of the forces exerted on the vehicle while driving.

TABle 1: Parameters in the vehicle longitudinal dynamics and braking strategy.

\begin{tabular}{lc}
\hline$m$ & Weight of the vehicle \\
$a_{\text {des }}$ & Desired acceleration \\
$C_{D}$ & Aerodynamic drag coefficient \\
$A$ & Automotive frontal area \\
$g$ & Gravity coefficient \\
$f$ & Coefficient of rolling resistance \\
$a$ & Road slope angle \\
$\delta$ & Rotational mass conversion coefficient \\
$R$ & Wheel radius \\
$i_{t}$ & Total transmission ratio \\
$\eta$ & Mechanical efficiency \\
$F_{t}$ & Motor driving force \\
$T_{t}^{d}$ & Desired driving torque \\
$T_{b}^{d}$ & Desired braking torque \\
$F_{b}^{d}$ & Desired braking force \\
$T_{r}^{d}$ & Desired regenerative braking torque \\
$T_{m}$ & Maximum braking torque of driving wheel \\
$z$ & Braking strength \\
SOC & State of charge \\
$v_{1}$ & Speed of the following vehicle \\
$p$ & Regenerative braking proportional coefficient \\
\hline
\end{tabular}

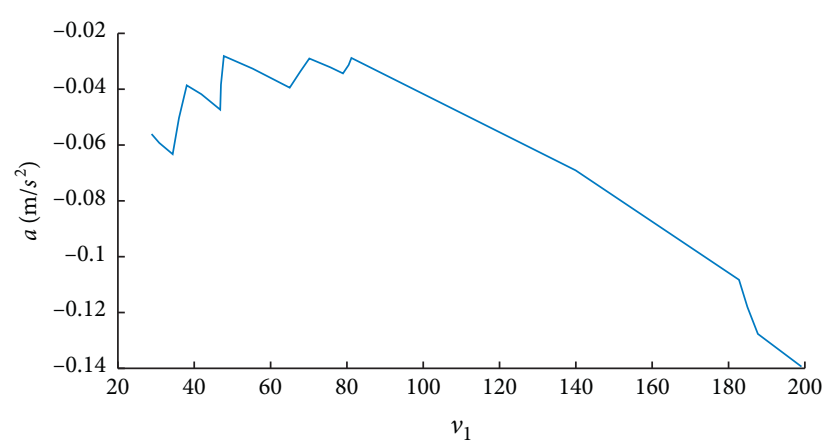

FIGURE 2: Ground deceleration of the following vehicle in a range of normal driving speed.

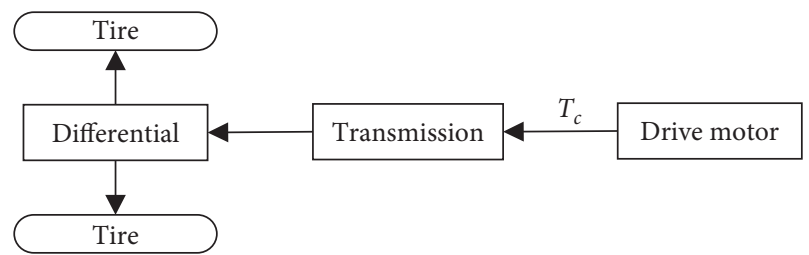

FIGURE 3: Schematic diagram of the vehicle powertrain system. 


$$
F_{b}^{d}=m z g
$$

Then, when SOC $>0.8$, in order to avoid battery overcharge, both front and rear wheels adopt mechanical braking; if SOC $\leq 0.8$, the form of braking depends on $z$ [25]. More precisely, given that $\mathrm{SOC} \leq 0.8, z<0.1$, regenerative braking works only, desired regenerative braking torque can be computed as

$$
T_{r}^{d}=T_{b}^{d}
$$

When SOC $\leq 0.8$ and $0.1<z<0.7$, the hybrid braking works, on the premise of ensuring safety, to increase the braking efficiency and recover braking energy as much as possible, Mamdani fuzzy control algorithm is used for the braking control considering the time variability and nonlinearity of the vehicle. In the hybrid braking, for the fuzzy logic controller, $z$, SOC, and $v_{1}$ are chosen as the inputs while $p$ is chosen as the output. The membership function curves of the inputs and output are presented in Figure 4 and parameters of the fuzzy logic controller are presented in Table $2[26,27]$.

The basic rules of regenerative braking are presented in Table 3 and detailed explanations are as follows:

(1) When SOC is large, $p$ is supposed to be small to prevent the battery from overcharging.

(2) When SOC is medium (or small), if $z$ is small, $p$ should be large to recycle the braking energy as much as possible; if $z$ is large, there should be a decrease in $p$ to provide sufficient braking force to ensure safety.

(3) When the safety is guaranteed, under the same condition, the larger $v_{1}$ is, the larger $p$ is, so as to recover braking energy fully; while the safety cannot be guaranteed, the larger $v_{1}$ is, the smaller the $p$ is to provide sufficient braking force.

Then, the desired regenerative braking torque is expressed as

$$
T_{r}^{d}=\min \left(p T_{b}^{d}, T_{m}\right)
$$

where $T_{m}$ is the maximum braking torque of the driving wheel limited by the motor characteristics and the road adhesion coefficient.

The fuzzy rules for the proportional coefficient of regenerative braking are presented in Table 4.

When $\mathrm{SOC} \leq 0.8, z>0.7$, mechanical braking works only to provide enough braking torque [28]. The braking torque distribution method when the front and rear wheels are locked simultaneously under ideal condition is adopted.

\section{Design of the Hybrid Controller in Longitudinal Dynamics Control and Lateral Dynamics Control}

In order to improve driving safety, this paper comprehensively considers the influence of the relative speed, the relative distance between the two vehicles, and the expected distance on the switching strategy. When $\Delta d>\Delta d_{\text {switch }}, v_{r}>v_{r_{-} \text {switch }}$, the ACC system switches to the cruising mode; otherwise, it switches to the following mode. $\Delta d=d-d_{\mathrm{des}}$ is the distance error; $d_{\mathrm{des}}$ is the expected distance calculated by the safety distance model; $v_{r}=v_{2}-v_{1}$.

The kinematic relations between the leading vehicle (LV) and the following vehicle (FV) are presented in Figure 5. Parameters in the longitudinal dynamics control are given in Table 5. The MPC algorithm based on SDNN and the PID controller based on $\mathrm{SN}$ are applied to the following mode and the cruising mode in the longitudinal dynamics control, respectively.

3.1. Incremental MPC Controller Based on SDNN in the Following Mode in Longitudinal Dynamics Control. When the ACC system switches into the following mode, the Incremental MPC controller based on SDNN works. The MPC model is derived from the kinematic relation. In order to establish a real-time system, $a_{1}$ is selected as the control variable, while $d, v_{r}$, and $v_{1}$ are selected as the state variables. The control variable and the state variable vector are as follows:

$$
\left\{\begin{array}{l}
u(k)=a_{1}(k), \\
\mathbf{x}(k)=\left(d(k), v_{r}(k), v_{1}(k)\right)^{T}
\end{array}\right.
$$

where $u(k)$ is the control variable and $\mathbf{x}(k)$ represents the state variables.

To simplify the prediction model, the acceleration of the $\mathrm{LV}$ is assumed to be $0[29,30]$. Then,

$$
a_{r}(k)=-a_{1}(k)
$$

Then, the simplified kinematics model is expressed as

$$
\left\{\begin{array}{l}
v_{1}(k+1)=v_{1}(k)+a_{1}(k) T_{s}, \\
d(k+1)=d(k)+v_{r}(k) T_{s}-\frac{1}{2} a_{1}(k) T_{s}^{2}, \\
v_{r}(k+1)=v_{r}(k)-a_{1}(k) T_{s} .
\end{array}\right.
$$

To prevent the acceleration from changing dramatically and improve the stability of the ACC system, $\Delta a_{1}$, the difference of acceleration between two adjacent sampling instants is selected as the nominal control variable of the system instead of the raw acceleration of the FV. It is expressed by the difference between the acceleration at the current sampling instant and the acceleration at the previous sampling instant as

$$
\Delta u(k)=u(k)-u(k-1)
$$

Then, (7) is rewritten as 


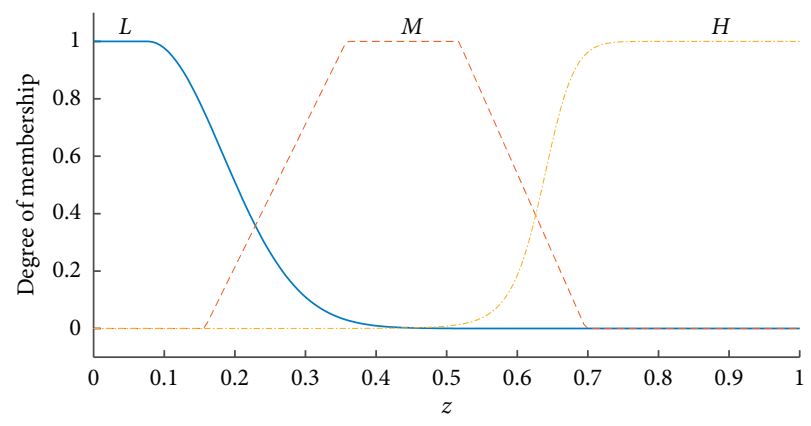

(a)

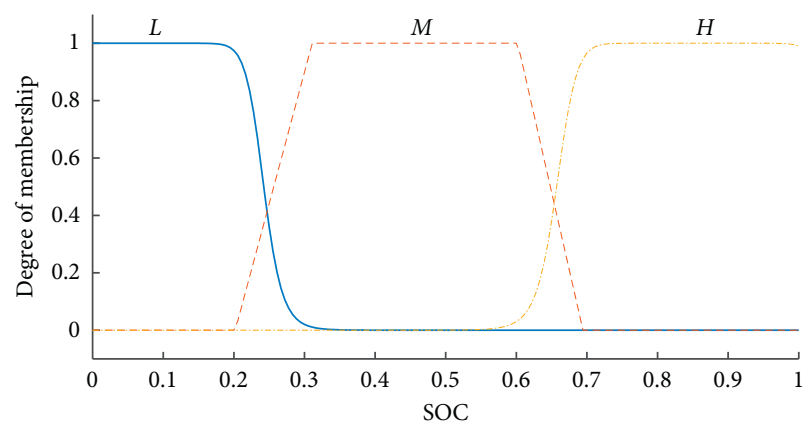

(c)

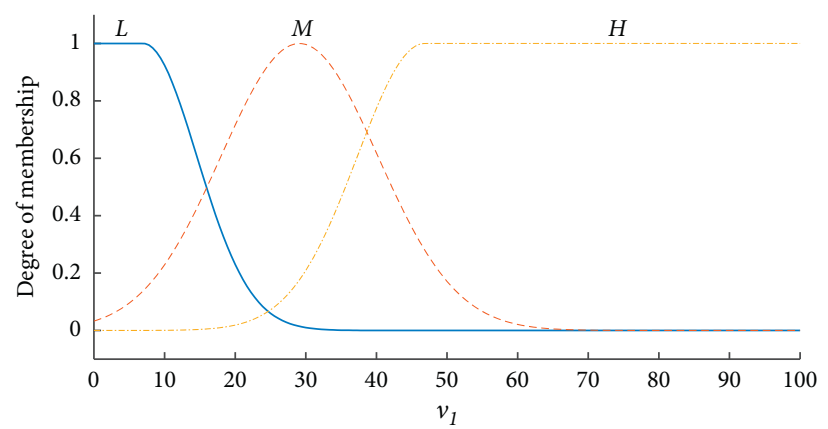

(b)

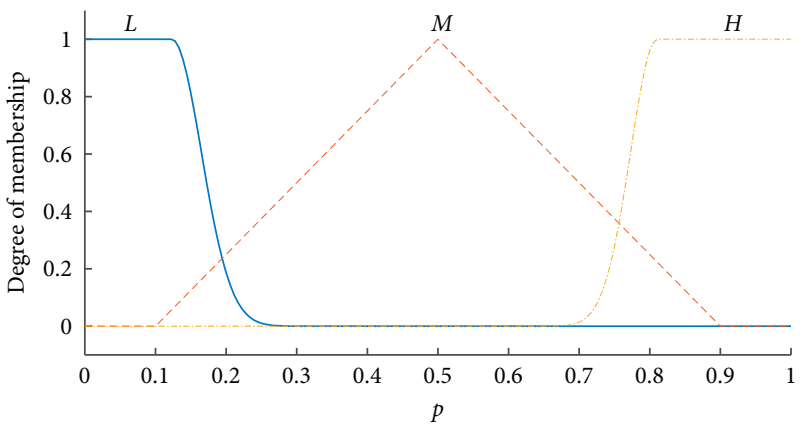

(d)

Figure 4: (a) The braking strength membership function curve. (b) The following-vehicle-speed membership function curve. (c) The membership function curve. (d) The driving wheel regenerative braking proportional coefficient membership function curve.

TABLE 2: Parameters of the fuzzy logic controller.

\begin{tabular}{lcc}
\hline Parameter & Theoretical domain & Fuzzy subset \\
\hline$v_{1}$ & $E_{z}=[L, M, H]$ & {$[0,100]$} \\
$z$ & $E_{z}=[L, M, H]$ & {$[0,1]$} \\
SOC & $E s o c=[L, M, H]$ & {$[0,1]$} \\
$p$ & $E p=[L, M, H]$ & {$[0,1]$} \\
\hline
\end{tabular}

$L, M, H$ represent low, medium, and high, respectively, in the fuzzy subsets.

TABLE 3: Basic rules of regenerative braking.

\begin{tabular}{lccc}
\hline$v_{1}$ & $z$ & SOC & $p$ \\
\hline- & $\uparrow$ & - & $\downarrow$ \\
$\uparrow$ & - & - & $\uparrow$ \\
\hline
\end{tabular}

$$
\left\{\begin{array}{l}
u^{\prime}(k)=u(k)-u(k-1), \\
\mathbf{x}^{\prime}(k)=\left(d(k), v_{r}(k), v_{1}(k), u(k-1)\right) .
\end{array}\right.
$$

The Incremental predictive equation of the state is

$$
\left\{\begin{array}{l}
\mathbf{x}^{\prime}(k+1 \mid k)=\mathbf{A} \mathbf{x}^{\prime}(k)+\mathbf{B} \mathbf{u}^{\prime}(k)+\mathbf{W}_{\mathbf{e}} \mathbf{e}(k), \\
\mathbf{y}^{\prime}(k)=\mathbf{C} \mathbf{x}^{\prime}(k),
\end{array}\right.
$$

where
TABLE 4: Fuzzy rules for the proportional coefficient of regenerative braking.

\begin{tabular}{lccc}
\hline SOC & $z$ & $v_{1}$ & $p$ \\
\hline$H$ & $L$ & $M$ & $L$ \\
$H$ & $L$ & $H$ & $L$ \\
$H$ & $L$ & $L$ & $L$ \\
$H$ & $M$ & $M$ & $L$ \\
$H$ & $M$ & $H$ & $L$ \\
$H$ & $M$ & $L$ & $L$ \\
$H$ & $H$ & $M$ & $L$ \\
$H$ & $H$ & $H$ & $L$ \\
$H$ & $H$ & $L$ & $L$ \\
$M$ & $L$ & $H$ & $H$ \\
$M$ & $L$ & $M$ & $H$ \\
$M$ & $L$ & $L$ & $L$ \\
$M$ & $M$ & $M$ & $M$ \\
$M$ & $M$ & $L$ & $L$ \\
$M$ & $M$ & $H$ & $M$ \\
$M$ & $H$ & $H$ & $L$ \\
$M$ & $H$ & $L$ & $L$ \\
$M$ & $H$ & $M$ & $L$ \\
$L$ & $L$ & $M$ & $H$ \\
$L$ & $L$ & $H$ & $H$ \\
$L$ & $L$ & $L$ & $L$ \\
$L$ & $M$ & $M$ & $M$ \\
$L$ & $M$ & $H$ & $M$ \\
$L$ & $M$ & $L$ & $L$ \\
$L$ & $H$ & $M$ & $L$ \\
$L$ & $H$ & $H$ & $L$ \\
$L$ & $H$ & $L$ &
\end{tabular}




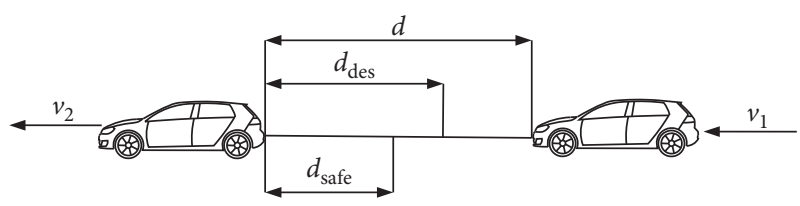

FIGURE 5: Scheme of the kinematic relations between the LV and the FV.

TABle 5: Parameters in the longitudinal dynamics controller design.

\begin{tabular}{|c|c|}
\hline$d$ & Relative longitudinal distance \\
\hline$d_{\mathrm{des}}$ & Desired longitudinal distance \\
\hline$d_{\text {safe }}$ & Longitudinal safety distance \\
\hline$\Delta d_{\mathrm{sw}}$ & Critical longitudinal distance error \\
\hline$v_{r}$ & Relative longitudinal speed \\
\hline$v_{r, \mathrm{sw}}$ & Critical relative longitudinal speed \\
\hline$v_{2}$ & Longitudinal speed of the following vehicle \\
\hline$a_{1}$ & Longitudinal acceleration of the following vehicle \\
\hline$a_{2}$ & Longitudinal acceleration of the leading vehicle \\
\hline$a_{r}$ & Relative longitudinal acceleration \\
\hline$\Delta r_{\min }$ & Low bound of longitudinal distance error \\
\hline$\Delta r_{\max }$ & Upper bound of longitudinal distance error \\
\hline$v_{r_{\min }}$ & Low bound of relative longitudinal speed error \\
\hline$v_{r_{\max }}^{\min }$ & Upper bound of relative longitudinal speed error \\
\hline$a_{r_{\min }}^{r_{\max }}$ & Low bound of longitudinal acceleration \\
\hline$a_{r_{\max }}^{\min }$ & Upper bound of longitudinal acceleration \\
\hline$v_{c}^{d^{\max }}$ & Desired cruising speed \\
\hline$T_{s}$ & Sampling time \\
\hline$N_{p}$ & Prediction time domain \\
\hline$N_{c}^{p}$ & Control time domain \\
\hline$\varepsilon$ & Relaxation factor \\
\hline$\rho$ & Undetermined coefficient \\
\hline$N_{x}$ & Number of the state variables \\
\hline$N_{u}$ & Number of the control variables \\
\hline$\Delta r_{\min }$ & Low bound of difference of longitudinal acceleration between two adjacent sampling instants \\
\hline$\Delta r_{\max }$ & Upper bound of difference of longitudinal acceleration between two adjacent sampling instants \\
\hline
\end{tabular}

$$
\begin{aligned}
\mathbf{A} & =\left(\begin{array}{cccc}
1 & T_{s} & 0 & -\frac{1}{2} T_{s}^{2} \\
0 & 1 & 0 & -T_{s} \\
0 & 0 & 1 & T_{s} \\
0 & 0 & 0 & 1
\end{array}\right) ; \\
\mathbf{B} & =\left(\begin{array}{c}
-\frac{1}{2} T_{s}^{2} \\
-T_{s} \\
T_{s} \\
1
\end{array}\right) ; \\
\mathbf{C} & =\operatorname{diag}(1,1,1,1) .
\end{aligned}
$$

and

$\mathbf{W}_{e}=\operatorname{diag}(0.025,0.025,0.025,0.025)$ $\mathbf{e}(k)=\mathbf{x}^{\prime}(k)-\mathbf{x}^{\prime}(k \mid(k-1))$ is the prediction error.

Then, the predicted state equation of the system can be expressed as

$$
\mathbf{Y}^{\prime}(k)=\boldsymbol{\psi} \mathbf{x}^{\prime}(k)+\boldsymbol{\theta} \Delta \mathbf{U}(k)+\mathbf{W}_{\mathrm{re}} \mathbf{e}(k),
$$

where

$$
\begin{aligned}
& \mathbf{Y}^{\prime}(k)=\left(\begin{array}{c}
\mathbf{y}^{\prime}(k+1) \mid k \\
\vdots \\
\mathbf{y}^{\prime}\left(k+N_{p}\right) \mid k
\end{array}\right) ; \\
& \psi=\left(\begin{array}{c}
\mathrm{CA} \\
\vdots \\
\mathrm{CA}^{N_{p}}
\end{array}\right) \\
& \boldsymbol{\theta}=\left(\begin{array}{cccc}
\mathbf{C B} & \cdots & 0 & 0 \\
\vdots & & \ddots & 0 \\
\mathbf{C A}^{N_{c}-1} \mathbf{B} & \cdots & \cdots & \mathbf{C B} \\
\vdots & & & \vdots \\
\mathbf{C A}^{N_{p}-1} \mathbf{B} & \cdots & \cdots & \sum_{i=1}^{N_{c}-N_{p}+1} \mathbf{C A}^{i-1} \mathbf{B}
\end{array}\right) \\
& \Delta \mathbf{U}(k)=\left(\begin{array}{c}
\Delta u(k) \\
\vdots \\
\Delta u\left(k+N_{c}-1\right)
\end{array}\right) ;
\end{aligned}
$$

$$
\mathbf{W}_{\mathrm{re}}=\left(\begin{array}{c}
\mathbf{C W}_{e} \\
\vdots \\
\mathbf{C A}^{N_{p}-1} \mathbf{W}_{e}
\end{array}\right) \text {. }
$$

In practical application, overall performances of tracking, safety, comfort, and energy economy should be taken 
into account to design an effective control strategy. Furthermore, the input, state, and output variables of the system should be constrained in a proper range to ensure that the ACC system normally runs. In the following mode, the ACC system should realize the function of following the LV on the premise of ensuring safety, improving comfort, and achieving better performances of energy economy and tracking. Therefore, the specific optimization goal is to reduce the tracking error, constrain the amplitude of $a_{1}$ and $\Delta a_{1}$, and restrain excessive oscillation. Hence, the following factors should be taken into account.

3.1.1. Energy Economy. The rapid acceleration and deceleration of the vehicle would increase the energy consumption, so it is necessary to limit the amplitude of $a_{1}$ and $\Delta a_{1}$. Therefore, this paper takes $a_{1}$ and $\Delta a_{1}$ as quantitative characteristic parameters of the economic indicator.

Define the economic indicator as

$$
J_{E} k+i \mid k=w_{a_{1}} a_{1}(k+i \mid k)^{2}+w_{\Delta a_{1}}\left(\Delta a_{1}(k+i \mid k)\right)^{2},
$$

where $w_{a_{1}}$ is the weight coefficient of $a_{1}$ and $w_{\Delta a_{1}}$ is the weight coefficient of $\Delta a_{1}, i=1,2, \ldots, N_{p}$.

3.1.2. Tracking. The ACC system is supposed to be able to control the FV to track the LV steadily in the following mode. On the one hand, the ACC system would control the relative distance between the two vehicles to approach the expected distance calculated by the safety distance model. On the other hand, it would also control the speed of the FV to approach the speed of the LV. While the LV and the FV are in a relatively stable driving state, the relative speed approaches 0 . This paper takes $\Delta d$ and $v_{r}$ between two vehicles as quantitative characteristic parameters of the tracking indicator.

Define the tracking indicator as

$$
\begin{aligned}
J_{F}((k+i) \mid k)= & w_{\Delta d}\left(d(k+i \mid k)-d_{\text {des }}(k+i \mid k)^{2}\right. \\
& +w_{v_{r}} v_{r}(k+i \mid k)^{2},
\end{aligned}
$$

and the constraints are

$$
\left\{\begin{array}{l}
\Delta d_{\min } \leq d(k+i \mid k)-d_{\text {des }}(k+i \mid k) \leq \Delta d_{\text {max }}, \\
v_{r \text { min }} \leq v_{r}(k+i \mid k) \leq v_{r \max },
\end{array}\right.
$$

where $w_{\Delta d}$ is the weight coefficient of $\Delta d$ and $w_{v_{r}}$ is the weight coefficient of $v_{r}, i=1,2, \ldots, N_{p}$.

3.1.3. Safety. To ensure safety of driving, the ACC system is expected to prevent rear-end collisions, so it is necessary to keep the relative longitudinal distance between the LV and the FV larger than the minimum longitudinal safety distance calculated by the safety distance model. Hence, $\Delta d$ between the LV and the FV is used as the quantitative characteristic parameter of the safety indicator.

The safety indicator is expressed as

$$
d(k+i \mid k)>d_{\text {safe }}(k+i) \mid k
$$

where $i=1,2, \ldots, N_{p}$.

3.1.4. Comfort. The comfort requirements of the ACC system can be summarized as follows:

(1) In order to avoid the frequent insertion of vehicles in adjacent lanes into the driving lane of the FV and keep enough safety distance from the LV to prevent discomfort to the driver, the relative distance and relative speed between the LV and the FV are supposed to meet the driver's expectation.

(2) The FV should keep the uniform speed as long as possible to avoid overlarge acceleration or deceleration.

(3) $a_{1}$ and $\Delta a_{1}$ of the FV should be within an appropriate range, so as to avoid causing discomfort to the occupants.

(1) and (2) have been reflected in the tracking indicator and the economic indicator, respectively. So, this paper takes $a_{1}$ and $\Delta a_{1}$ as quantitative characteristic parameters of the comfort indicator and limits the range of them.

The comfort indicator is formulated as

$$
\left\{\begin{array}{l}
a_{\min } \leq a_{1}(k+i \mid k) \leq a_{\max } \\
\Delta a_{\min } \leq \Delta a_{1}(k+i \mid k) \leq \Delta a_{\max }
\end{array}\right.
$$

where $i=1,2, \ldots, N_{p}$.

3.1.5. Comprehensive Performance Function. The above indicators only consider a single factor respectively, and a comprehensive performance function is needed to evaluate the overall performance of the ACC system. Therefore, the combinations of the economic indicator, the tracking indicator, the safety indicator, and the comfort indicator are taken as the comprehensive performance function. And each constraint in the individual indicator is taken as the constraint of the comprehensive performance function. Then, the comprehensive performance function is expressed as

$$
\begin{aligned}
J((k+i) \mid k)= & J_{E}(k+i \mid k)+J_{F}(k+i \mid k) \\
= & w_{a_{1}} a_{1}(k+i \mid k)^{2}+w_{\Delta a_{1}}\left(\Delta a_{1}(k+i \mid k)\right)^{2} \\
& +w_{d}\left(d(k+i \mid k)-d_{\mathrm{des}}(k+i \mid k)\right)^{2} \\
& +w_{v_{r}} v_{r}(k+i \mid k)^{2} .
\end{aligned}
$$

Put $\quad \mathbf{y}^{\prime}((k+i) \mid k)=\left(d((k+i) \mid k), v_{r}((k+i) \mid k), v_{1}\right.$ $((k+i) \mid k), u(k+i-1))^{T}$ into $(21)$ and replace $a_{1}, \Delta a_{1}$ with $u$ and $\Delta u$. Considering the prediction time domain is $N_{p},(21)$ is transferred into

$$
\begin{aligned}
& J((k+i) \mid k)=\left(\mathbf{y}^{\prime}((k+i) \mid) k-\mathbf{y}_{\text {ref }}^{\prime}((k+i) \mid k)\right)^{T} \mathbf{w}_{\mathbf{y}} \\
& \quad\left(\mathbf{y}^{\prime}((k+i) \mid k)-\mathbf{y}_{\text {ref }}^{\prime}((k+i) \mid k)\right)+w_{\Delta a_{h}}(\Delta u(k+i-1))^{2},
\end{aligned}
$$

where 


$$
\begin{aligned}
\mathbf{w}_{\mathbf{y}} & =\left(\begin{array}{cccc}
w_{d} & 0 & 0 & 0 \\
0 & w_{v_{r}} & 0 & 0 \\
0 & 0 & 0 & 0 \\
0 & 0 & 0 & w_{a_{1}}
\end{array}\right) ; \\
\mathbf{y}_{\text {ref }}^{\prime}((k+i) \mid k) & =\left(\begin{array}{c}
d_{\text {des }}((k+i) \mid k) \\
0 \\
v_{2}((k+i) \mid k) \\
0
\end{array}\right) ; \quad i=1,2, \ldots, N_{p} .
\end{aligned}
$$

In the prediction time domain, the prediction equation of the comprehensive performance function is as follows:

$$
\begin{aligned}
J= & \sum_{i=1}^{N_{p}} J((k+i) \mid k)=\sum_{i=1}^{N_{p}} J_{E}((k+i) \mid k)+\sum_{i=1}^{N_{p}} J_{F}((k+i) \mid k) \\
= & \left.\sum_{i=1}^{N_{p}}\left(\mathbf{y}^{\prime}((k+i) \mid) k\right)-\mathbf{y}_{\text {ref }}^{\prime}((k+i) \mid k)\right)^{T} \mathbf{w}_{\mathbf{y}} \\
& \left(\mathbf{y}^{\prime}((k+i) \mid k)-\mathbf{y}_{\text {ref }}^{\prime}((k+i) \mid k)\right)+w_{\Delta a_{1}}(\Delta u(k+i-1))^{2} \\
= & \sum\left\|\mathbf{y}^{\prime}-\mathbf{y}_{\text {ref }}^{\prime}\right\|_{Q}^{2}+\sum\|\Delta u\|_{R}^{2} \\
= & \left(\mathbf{Y}^{\prime}-\mathbf{Y}_{\text {ref }}^{\prime}\right)^{T} \mathbf{Q}\left(\mathbf{Y}^{\prime}-\mathbf{Y}_{\text {ref }}^{\prime}\right)+\Delta \mathbf{U}^{T} \mathbf{R} \Delta \mathbf{U},
\end{aligned}
$$

where

$$
\begin{aligned}
\mathbf{Q} & =\mathbf{I}_{N_{p}} \otimes \mathbf{w}_{\mathbf{y}} ; \\
\mathbf{R} & =\mathbf{I}_{N_{p}} \otimes w_{\Delta a_{1}} ; \\
\mathbf{Y}^{\prime} & =\left(\begin{array}{c}
\mathbf{y}^{\prime}(k+1) \\
\vdots \\
\mathbf{y}^{\prime}\left(k+N_{p}\right)
\end{array}\right) ; \\
\mathbf{Y}_{\mathrm{ref}}^{\prime} & =\left(\begin{array}{c}
\mathbf{y}_{\mathrm{ref}}^{\prime}(k+1) \\
\vdots \\
\mathbf{y}_{\mathrm{ref}}^{\prime}\left(k+N_{p}\right) \\
\Delta u(k) \\
\vdots \\
\Delta \mathbf{U}
\end{array}=\left(\begin{array}{c} 
\\
\Delta u\left(k+N_{c}-1\right)
\end{array}\right) .\right.
\end{aligned}
$$
as

The predictive optimization problem can be summarized

$\min J(\mathbf{Y}, \Delta \mathbf{U})$

$$
\text { s.t. }\left\{\begin{array}{l}
\max \left(d_{\text {safe }}, \Delta d_{\min }+d_{\mathrm{des}}\right) \leq d(k+i \mid k) \leq \Delta d_{\max }+d_{\mathrm{des}} \\
v_{r \min } \leq v_{r}(k+i \mid k) \leq v_{r \max } \\
u_{\min } \leq u(k+i \mid k) \leq u_{\max } \\
\Delta u_{\min } \leq \Delta u(k+j \mid k) \leq \Delta u_{\max }
\end{array}\right.
$$

where $i=1,2, \ldots, N_{p}$ and $j=0,1,2, \ldots, N_{c}$.

Transform (26) into a QP problem for solving as

$$
\begin{aligned}
J^{\prime}= & \sum\left\|\mathbf{y}^{\prime}-\mathbf{y}_{\mathrm{ref}}{ }^{\prime}\right\|_{\mathbf{Q}}^{2}+\sum\|\Delta u\|_{\mathbf{R}}^{2}+\rho \varepsilon^{2} \\
= & \left(\boldsymbol{\psi} \mathbf{x}^{\prime}(k)-\mathbf{Y}_{\mathrm{ref}}{ }^{\prime}+\boldsymbol{\theta} \Delta \mathbf{U}+\mathbf{W}_{\mathrm{re}} \mathbf{e}(k)\right)^{T} \\
& \mathbf{Q}\left(\boldsymbol{\psi} \mathbf{x}^{\prime}(k)-\mathbf{Y}_{\mathrm{ref}}{ }^{\prime}+\boldsymbol{\theta} \Delta \mathbf{U}+\mathbf{W}_{\mathrm{re}} \mathbf{e}(k)\right)+\Delta \mathbf{U}^{T} \mathbf{R} \Delta \mathbf{U}+\rho \varepsilon^{2} \\
= & \cdots \\
= & \left(\begin{array}{ll}
\Delta \mathbf{U}^{T} & \varepsilon
\end{array}\right)\left(\begin{array}{cc}
\boldsymbol{\theta}^{T} \mathbf{Q} \boldsymbol{\theta}+\mathbf{R} & 0 \\
0 & \rho
\end{array}\right)\left(\begin{array}{c}
\Delta \mathbf{U} \\
\varepsilon
\end{array}\right)+\left(\begin{array}{ll}
2 \mathbf{E}_{1}^{T} & 0
\end{array}\right)\left(\begin{array}{c}
\Delta \mathbf{U} \\
\varepsilon
\end{array}\right) \\
= & \frac{1}{2} \zeta^{T} \mathbf{H} \boldsymbol{\varepsilon}+\mathbf{f}^{T} \zeta,
\end{aligned}
$$

where

$$
\begin{aligned}
\mathbf{E}_{1} & =\boldsymbol{\psi} \mathbf{x}^{\prime}(k)-\mathbf{Y}_{\mathrm{ref}}^{\prime}+\mathbf{W}_{\mathrm{Re}} \mathbf{e}(k) ; \\
\mathbf{H} & =\left(\begin{array}{cc}
2 \boldsymbol{\theta}^{T} \mathbf{Q} \boldsymbol{\theta}+2 \mathbf{R} & 0 \\
0 & 2 \rho
\end{array}\right) ; \\
\mathbf{f}^{T} & =\left(\begin{array}{cc}
2 \mathbf{E}_{1}^{T} & 0
\end{array}\right) ; \\
\boldsymbol{\zeta} & =\left(\begin{array}{c}
\Delta \mathbf{U} \\
\varepsilon
\end{array}\right) .
\end{aligned}
$$

The feasibility of MPC is enhanced by softening nonsafety related hard constraints. The predictive optimization problem is transformed to the standard quadratic form for the solution by the Quadprog function. However, its realtime performance is poor and not applicable to the actual embedded system. So, this paper uses SDNN to accelerate the solution of the QP problem which is an innovation compared to other research studies.

The state equation of SDNN is

$$
\xi \frac{\mathrm{d} \mathbf{z}}{\mathrm{d} t}=-\mathbf{E D z}+(\mathbf{E D}-\mathbf{I}) g(\mathbf{z})-\mathbf{E s}
$$

The principle of SDNN is presented in Figure 6. $z_{i}$ is the neuron, $g(\mathbf{z})$ is a nonlinear activation function, ED - I is the symmetric weight matrix, and SDNN can be seen as a monolayer periodic neural network model. $g(\mathbf{z})=\left(g\left(z_{1}\right), g\left(z_{2}\right), g\left(z_{3}\right), \ldots, g\left(z_{p}\right)\right)^{T}$ is a piecewise linear function, which is expressed as

$$
\begin{aligned}
g\left(z_{i}\right) & =\left\{\begin{array}{l}
l_{i} z_{i} \leq l_{i}, \\
z_{i} l_{i}<z_{i}<l_{i}, \\
h_{i} z_{i} \geq h_{i},
\end{array}\right. \\
\mathbf{z} & =\mathbf{E x}_{\mathbf{Q P}}-\mathbf{v}, \\
\mathbf{x}_{\mathbf{Q P}} & =\mathbf{D} \mathbf{v}-\mathbf{s},
\end{aligned}
$$

where $\mathbf{v}$ is the state variable and $\xi(>0)$ is the scaling factor that regulates the convergence rate of the SDNN model. 


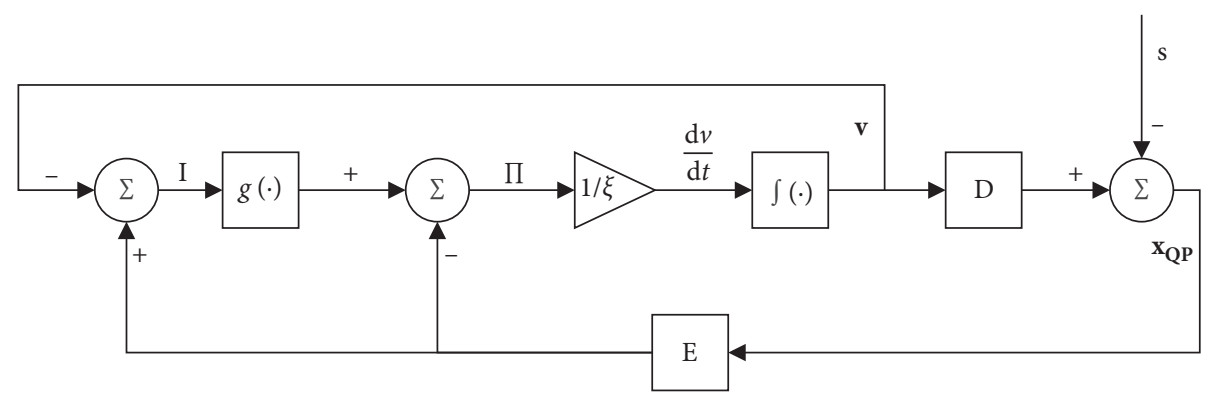

FIgURE 6: Principle block diagram of SDNN [31].

Compared with the conventional numerical solution, the calculation in SDNN does not involve matrix inversion, decomposition, and other complex operations. So, the computational complexity to solve QP by SDNN is lower. Furthermore, SDNN occupies fewer resources and the algorithm is more convenient to implement. And Liu [31] has proved that SDNN globally convergences to the optimal solution of the QP problem in the form of (27). In order to solve the QP problem by computer, SDNN has to be discretized. This paper uses the Euler method to discrete (29); then,

$$
\mathbf{z}(k+1)=\frac{1}{\xi} T_{\text {step }}(-\mathbf{E D z}(k)+(\mathbf{E D}-\mathbf{I}) g(\mathbf{z}(k))-\mathbf{E s})+\mathbf{z}(k)
$$

Considering the QP problem in (27), the corresponding relationship between the discrete SDNN parameters and quadratic programming parameters can be expressed as

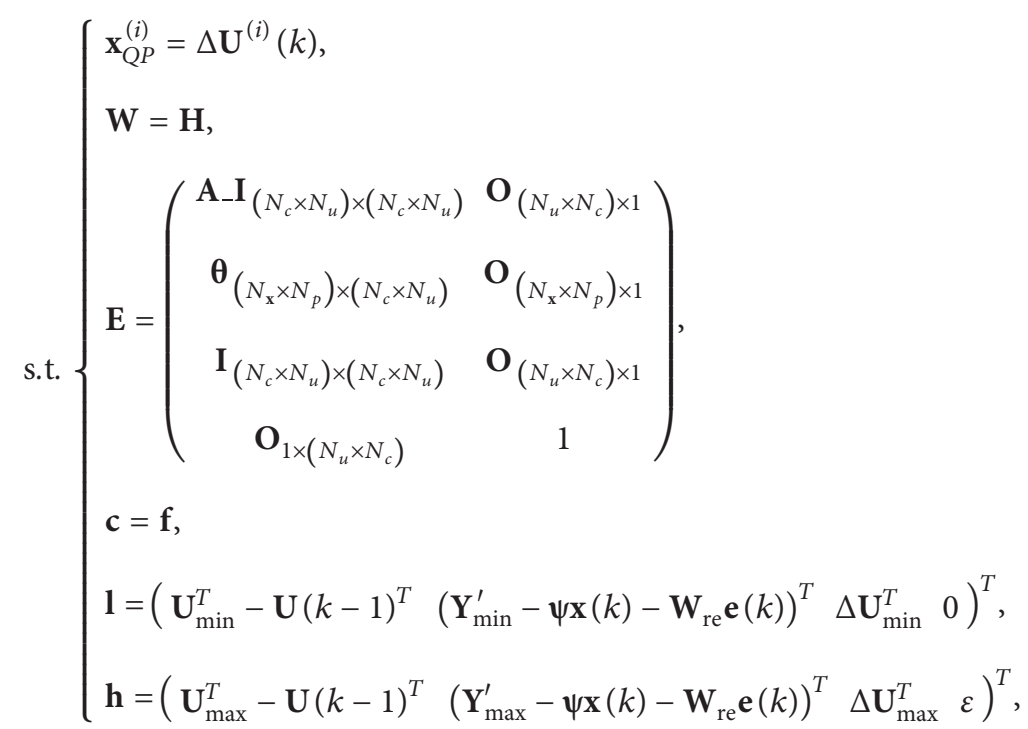

where 


$$
\begin{aligned}
& \mathbf{A}_{-} \mathbf{I}_{\left(N_{c} \times N_{u}\right) \times\left(N_{c} \times N_{u}\right)}=\left(\begin{array}{cccc}
0 & 0 & \cdots & 0 \\
1 & 0 & \ddots & 0 \\
1 & \vdots & \vdots & 0 \\
1 & 1 & 1 & 1
\end{array}\right)_{\left(N_{c} \times N_{u}\right) \times\left(N_{c} \times N_{u}\right)} \\
& \mathbf{y}_{\min }^{\prime}=\left(\begin{array}{c}
\max \left(d_{\mathrm{safe}}, \Delta d_{\min }+d_{\mathrm{des}}\right) \\
v_{r \min } \\
v_{2}-v_{r \max } \\
u_{\min }
\end{array}\right) \\
& \mathbf{y}_{\max }^{\prime}=\left(\begin{array}{c}
\Delta d_{\max }+d_{\mathrm{des}} \\
v_{r \max } \\
v_{2}-v_{r \min } \\
u_{\max }
\end{array}\right) \text {; } \\
& \mathbf{U}(k-1)=\left(\begin{array}{c}
u(k-1) \\
\vdots \\
u(k-1)
\end{array}\right)_{N_{c} \times 1} ; \\
& \mathbf{U}_{\min }=\left(\begin{array}{c}
u_{\min } \\
\vdots \\
u_{\min }
\end{array}\right)_{N_{c} \times 1} ; \\
& \mathbf{U}_{\max }=\left(\begin{array}{c}
u_{\max } \\
\vdots \\
u_{\max }
\end{array}\right)_{N_{c} \times 1} ; \\
& \Delta \mathbf{U}_{\min }=\left(\begin{array}{c}
\Delta u_{\min } \\
\vdots \\
\Delta u_{\min }
\end{array}\right)_{N_{c} \times 1} ; \\
& \Delta \mathbf{U}_{\max }=\left(\begin{array}{c}
\Delta u_{\max } \\
\vdots \\
\Delta u_{\max }
\end{array}\right)_{N_{c} \times 1} ; \\
& \mathbf{Y}_{\min }^{\prime}=\left(\begin{array}{c}
\mathbf{y}_{\min }^{\prime} \\
\vdots \\
\mathbf{y}_{\min }^{\prime}
\end{array}\right)_{N_{p} \times 1} ; \\
& \mathbf{Y}_{\max }^{\prime}=\left(\begin{array}{c}
\mathbf{y}_{\max }^{\prime} \\
\vdots \\
\mathbf{y}_{\max }^{\prime}
\end{array}\right)_{N_{p} \times 1} ; \\
& \mathbf{D}=\mathbf{W}^{-1} \mathbf{E}^{T} \text {; } \\
& s=\mathbf{W}^{-1} \mathbf{c} \text {. }
\end{aligned}
$$

The implementation of the SDNN algorithm is given in Algorithm 1.

3.2. PID Controller Based on Single Neuron in the Cruising Mode in Longitudinal Dynamics Control. When the ACC system switches into the cruising mode, the PID controller based on SN takes effect. Theoretically, single neuron can approach any nonlinear function infinitely, which ensures robustness and better performance while cruising, so the PID controller based on $\mathrm{SN}$ is designed for the cruising mode in this paper.

Desired cruising speeds $v_{c}^{d}$ and $v_{1}$ are seen as $\mathbf{y}_{r}(k)$ and $\mathbf{y}(k)$ in Figure 7 , and they are taken as the inputs, while $u(k)$ is set to be $a_{1} ; \mathbf{x}_{1}, \mathbf{x}_{2}, \mathbf{x}_{3}$ are the state variables required for neuronal learning control and taken as outputs of the converter; the vehicle model is seen as the plant. The adaptive PID controller based on SN designed in this paper is based on the Incremental PID, and the equations are

$$
\left\{\begin{array}{l}
\mathbf{x}_{1}=\mathbf{y}_{r}(k)-\mathbf{y}(k)=e(k), \\
\mathbf{x}_{2}=e(k)-e(k-1), \\
\mathbf{x}_{3}=e(k)-2 e(k-1)+e(k-2) .
\end{array}\right.
$$

The structure of the PID controller based on SN is presented in Figure 7. $z(k)=\mathbf{y}_{r}(k)-\mathbf{y}(k)=e(k)$ is the performance index; $K>0$ is the proportional coefficient of neurons. The equation of control signal $u(k)$ is

$$
u(k)=u(k-1)+K \sum_{i=1}^{3} w_{i}(k) \mathbf{x}_{i}(k),
$$

where $w_{i}(k)$ is the corresponding weight coefficient of $\mathbf{x}_{i}(k)$.

Then, the single-neuron-based PID control algorithm is as follows:

$$
\left\{\begin{array}{l}
\Delta e(k)=e(k)-e(k-1), \\
w_{1}(k)=w_{1}(k-1)+\eta_{I} z(k) u(k)(e(k)+\Delta e(k)), \\
w_{2}(k)=w_{2}(k-1)+\eta_{P} z(k) u(k)(e(k)+\Delta e(k)), \\
w_{3}(k)=w_{3}(k-1)+\eta_{D} z(k) u(k)(e(k)+\Delta e(k)), \\
w_{i}(k)=\frac{w_{j}(k)}{\sum_{j=1}^{3}\left|w_{j}(k)\right|}, \\
u(k)=u(k-1)+K \sum_{i=1}^{3} w_{i}(k) \mathbf{x}_{i}(k),
\end{array}\right.
$$

where $\eta_{I}, \eta_{P}$, and $\eta_{D}$ are the learning rates of the integration, proportion, and differentiation, respectively.

3.3. Lateral Dynamics Control. Most ACC systems in the literature are designed for longitudinal dynamics control and ignore lateral dynamics control; therefore, they cannot work on the curved road. In contrast, one contribution of this paper is taking lateral dynamics control into consideration; hence, the proposed ACC system can work on the curved road.

On the basis of (9) of the longitudinal kinematic model, we combine it with lateral dynamics control in 3-DoF vehicle 
(1) $k=0$. Determine whether to initialize; if yes, step to 3; if no, step 2;

(2) Update the status variables and the output variables, feedback correction;

(3) Set the iteration instant $i=1$ of discrete SDNN iteration to solve QP;

(4) Discrete SDNN iterates to solve the QP problem;

(a) Calculate $\Delta \mathbf{U}^{(i)}(k)$ of the current iteration instant according to discrete SDNN model;

(b) Calculate the relative error;

$$
\begin{aligned}
& \text { error }= \begin{cases}\left\|\Delta \mathbf{U}^{(i)}(k)-\Delta \mathbf{U}^{(i-1)}(k)\right\| /\left\|\Delta \mathbf{U}^{(i-1)}(k)\right\|, & \left(\left\|\Delta \mathbf{U}^{(i-1)}(k)\right\| \neq 0\right), \\
\left\|\Delta \mathbf{U}^{(i)}(k)-\Delta \mathbf{U}^{(i-1)}(k)\right\|, & \left(\left\|\Delta \mathbf{U}^{(i-1)}(k)\right\|=0\right)\end{cases} \\
& \text { Where } \\
& \Delta \mathbf{U}^{(0)}(k)=\mathbf{D v}^{(0)}-s ;
\end{aligned}
$$

(c) Determine whether error $\leq \varepsilon_{\mathrm{QP}}$, if yes, step to 5; if no, step to d);

(d) $i=i+1$, step to a);

(5) Get instant control increment $\Delta u(k)$, calculate the actual control variable $u(k)$.

(6) $k=k+1$, step to 2

Algorithm 1: Algorithm implementation of SDNN.

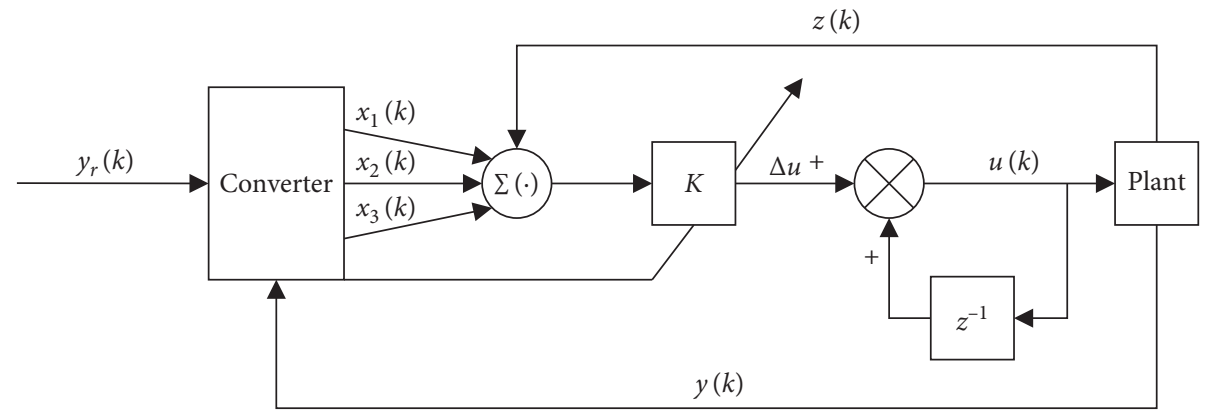

FIGURE 7: Structure block diagram of the PID controller based on SN.

model as shown in Figure 8. Parameters in the lateral dynamics control are given in Table 6.

In the lateral dynamics control, the nonlinear dynamics model is formed as $\dot{\boldsymbol{\xi}}_{\text {dyn }}(t)=f_{\text {dyn }}\left(\dot{\boldsymbol{\xi}}_{\text {dyn }}, \dot{u}_{\text {dyn }}\right)$. The state vector is $\boldsymbol{\xi}_{\mathrm{dyn}}=[\dot{\mathbf{y}}, \dot{\mathbf{x}}, \varphi, \dot{\varphi}, \mathbf{Y}, X]^{T} ; u_{\mathrm{dyn}}=\delta_{f}$ is chosen as the control variable [32]. $\dot{\mathbf{y}}, \dot{\mathbf{x}}$ are $v_{\mathbf{y}}$ and $v_{\mathbf{x}}$ in Figure 8 , respectively, and $v_{\mathbf{x}}$ is computed in the longitudinal dynamics control. Then, the nonlinear dynamic model can be linearized as

$$
\dot{\boldsymbol{\xi}}_{\mathrm{dyn}}(t)=\mathbf{A}_{\mathrm{dyn}}(t) \boldsymbol{\xi}_{\mathrm{dyn}}(t)+\mathbf{B}_{\mathrm{dyn}}(t) u_{\mathrm{dyn}}(t),
$$

where

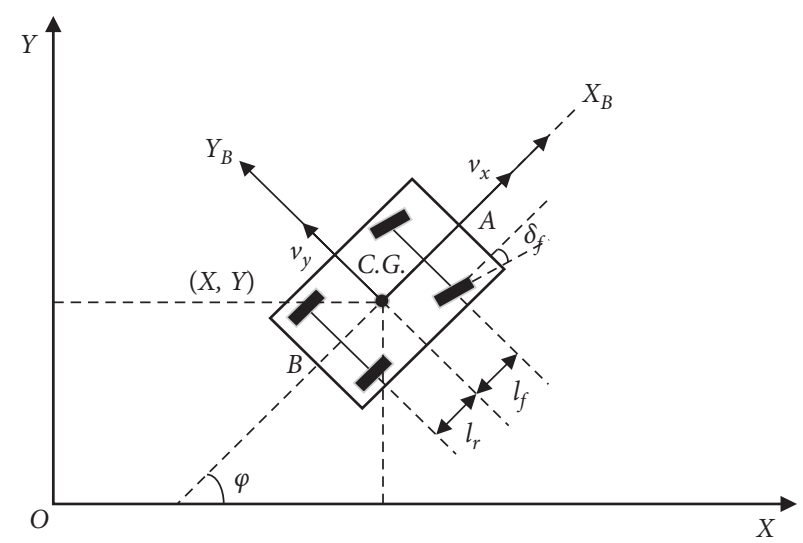

FIgURe 8: 3-DoF vehicle model. 
Table 6: Parameters in the lateral dynamics controller design.

\begin{tabular}{lc}
\hline$\dot{y}$ & Lateral velocity of vehicle in the body-fixed coordinate system \\
$\dot{\varphi}$ & Yaw rate of vehicle \\
$X$ & Abscissa of C.G. of vehicle \\
$I_{z}$ & Moment of inertia around the vertical axis through C.G. \\
$l_{r}$ & Distance from point B to point C.G. \\
$C_{\mathrm{cr}}$ & Lateral stiffness coefficients of rear tires \\
$C_{\mathrm{lr}}$ & Lateral stiffness coefficients of front tires \\
$\Delta u_{\max }^{\prime}$ & Upper bound of lateral motion \\
$Y_{\max }$ & Upper bound of lateral motion \\
$\varphi_{\max }$ & Lower bound of steering angle \\
$u_{\min }^{\prime}$ & Upper bound of difference of steering angle between two adjacent sampling instants \\
$\psi$ & Ordinate of $C . G$. of vehicle \\
$Y$ & Front steering angle \\
$\delta_{f}$ & Angle from $x$-axis to longitudinal axis of vehicle body \\
$l_{f}$ & Distance from point A to point $C . G$. \\
$C_{\mathrm{cf}}$ & Lateral stiffness coefficients of front tires \\
$C_{\mathrm{lf}}$ & Longitudinal stiffness coefficients of front tires \\
$\Delta u_{\min }^{\prime}$ & Low bound of lateral motion \\
$Y_{\min }$ & Low bound of lateral motion \\
$\varphi_{\min }$ & Upper bound of steering angle \\
$u_{\max }^{\prime}$ &
\end{tabular}

$$
\begin{aligned}
& \mathrm{B}_{\mathrm{dyn}}(t)=\left.\frac{\partial f_{\mathrm{dyn}}}{\partial u_{\mathrm{dyn}}(t)}\right|_{\xi_{t}, u_{t}}=\left[\frac{2 C_{\mathrm{cf}}}{m}, \frac{2 C_{\mathrm{cf}}\left(2 \delta_{f, t-1}-\left(\left(\dot{\mathbf{y}}_{t}+l_{f} \dot{\varphi}_{t}\right) / \dot{\mathbf{x}}_{t}\right)\right)}{m}, 0, \frac{2 l_{f} C_{\mathrm{cf}}}{m}, 0,0\right] \\
& \mathbf{A}_{\mathrm{dyn}}(t)=\left.\frac{\partial f_{\mathrm{dyn}}}{\partial \xi_{\mathrm{dyn}}}\right|_{\xi_{t}, \mathbf{u}_{t}} \\
& {\left[\begin{array}{cccccc}
\frac{-2\left(C_{\mathrm{cf}}+C_{\mathrm{cr}}\right)}{m \dot{\mathbf{x}}_{t}} & \frac{\partial f_{\dot{\mathbf{y}}}}{\partial \dot{\mathbf{x}}} & 0 & -\dot{\mathbf{x}}_{t}+\frac{2\left(l_{r} C_{\mathrm{cr}}-l_{f} C_{\mathrm{cf}}\right)}{m \dot{\mathbf{x}}_{t}} & 0 & 0 \\
\dot{\varphi}-\frac{2 C_{\mathrm{cf}} \delta_{f, t-1}}{m \dot{\mathbf{x}}_{t}} & \frac{\partial f_{\dot{\mathbf{x}}}}{\partial \dot{\mathbf{x}}} & 0 & \dot{\mathbf{y}}_{t}-\frac{2 l_{r} C_{\mathrm{cf}} \delta_{f, t-1}}{m \dot{\mathbf{x}}_{t}} & 0 & 0 \\
0 & 0 & 0 & -2\left(l_{f}^{2} C_{\mathrm{cf}}+l_{r}^{2} C_{\mathrm{cr}}\right) & 0 & 0 \\
\frac{I_{z} \dot{\mathbf{x}}_{t}}{2\left(l_{r} C_{\mathrm{cr}}-l_{f} C_{\mathrm{cf}}\right)} & \frac{\partial f_{\varphi}}{\partial \dot{\mathbf{x}}_{t}} & 0 & 0 & 0 & 0 \\
\cos \left(\varphi_{t}\right) & \sin \left(\varphi_{t}\right) & \dot{\mathbf{x}}_{t} \cos \left(\varphi_{t}\right)-\dot{\mathbf{y}}_{t} \sin \left(\varphi_{t}\right) & 0 & 0 & 0 \\
-\sin \left(\varphi_{t}\right) & \cos \left(\varphi_{t}\right) & -\dot{\mathbf{y}}_{t} \cos \left(\varphi_{t}\right)-\dot{\mathbf{x}}_{t} \sin \left(\varphi_{t}\right) & 0 & 0
\end{array}\right]} \\
& \frac{\partial f_{\dot{\mathbf{y}}}}{\partial \dot{\mathbf{x}}}=\frac{\left(2 C_{\mathrm{cf}}\left(\dot{\mathbf{y}}_{t}+l_{f} \dot{\varphi}_{t}\right)+2 C_{\mathrm{cr}}\left(\dot{\mathbf{y}}_{t}-l_{r} \dot{\varphi}_{t}\right)\right)}{m \dot{\mathbf{x}}_{t}^{2}}-\dot{\varphi}_{t} ; \\
& \frac{\partial f_{\dot{\mathbf{x}}}}{\partial \dot{\mathbf{x}}}=\frac{\left(2 C_{\mathrm{cf}} \delta_{f, t-1}\left(\dot{\mathbf{y}}_{t}+l_{f} \dot{\varphi}_{t}\right)\right)}{m \dot{\mathbf{x}}_{t}^{2}} \\
& \frac{\partial f_{\dot{\varphi}}}{\partial \dot{\mathbf{x}}}=\frac{\left(2 C_{\mathrm{cf}} l_{f}\left(\dot{\mathbf{y}}_{t}+l_{f} \dot{\varphi}_{t}\right)-2 C_{\mathrm{cr}} l_{r}\left(\dot{\mathbf{y}}_{t}-l_{r} \dot{\varphi}_{t}\right)\right)}{I_{z} \dot{\mathbf{x}}_{t}^{2}} .
\end{aligned}
$$


Then, (37) is discretized by the first-order Euler difference method, and the discrete state space is expressed as

$$
\boldsymbol{\xi}_{\text {dyn }}(k+1)=\widetilde{\mathbf{A}}_{\text {dyn }}(k) \boldsymbol{\xi}_{\text {dyn }}(k)+\widetilde{\mathbf{B}}_{\text {dyn }}(k) u_{\text {dyn }}(k),
$$

where $\widetilde{\mathbf{A}}_{\mathrm{dyn}}(k)=I+T_{s} \mathbf{A}_{\mathrm{dyn}}(t)$ and $\widetilde{\mathbf{B}}_{\mathrm{dyn}}(k)=T_{s} \widetilde{\mathbf{B}}_{\mathrm{dyn}}(t)$.

Then, the constraints of lateral motion are as follows:

$$
\begin{gathered}
\varphi_{\min } \leq \varphi \leq \varphi_{\max }, \\
\mathbf{Y}_{\min } \leq \mathbf{Y} \leq \mathbf{Y}_{\max } .
\end{gathered}
$$

In the prediction time domain, the prediction equation of the performance function of lateral motion is

$$
\begin{aligned}
J= & \sum_{i=1}^{N_{p}}\left\|\boldsymbol{\eta}_{\text {dyn }}(k+i \mid k)-\boldsymbol{\eta}_{\text {dyn,ref }}(k+i \mid k)\right\|_{Q_{1}}^{2} \\
& +\sum_{i=1}^{N_{c}}\left\|u_{\text {dyn }}(k+i \mid k)\right\|_{R_{1}}^{2}+\rho \varepsilon^{2},
\end{aligned}
$$

where $\boldsymbol{\eta}_{\text {dyn }}=[0,0,1,0,1,0] \cdot \boldsymbol{\xi}_{\text {dyn }}=[\varphi, \mathbf{Y}]$.

The predictive optimization problem can be summarized as

$$
\begin{aligned}
& \min \sum_{i=1}^{N_{p}}\left\|\mathbf{\eta}_{\text {dyn }}(k+i \mid k)-\boldsymbol{\eta}_{\text {dyn, ref }}(k+i \mid k)\right\|_{\mathbf{Q}_{1}}^{2} \\
& +\sum_{i=1}^{N_{c}}\left\|u_{\text {dyn }}(k+i \mid k)\right\|_{\mathbf{R}_{1}}^{2}+\rho \varepsilon^{2}, \\
& \text { s.t. }\left\{\begin{array}{l}
u_{\min } \leq \Delta u(k+i \mid k) \leq u_{\max }, \\
\Delta u_{\min }^{\prime} \leq u(k+i \mid k)-u((k+i-1) \mid k) \leq \Delta u_{\min }^{\prime}, \\
\mathbf{Y}_{\min } \leq \mathbf{Y}(k+i \mid k) \leq \mathbf{Y}_{\max }, \\
\varphi_{\min } \leq \varphi(k+i \mid k) \leq \varphi_{\max },
\end{array}\right.
\end{aligned}
$$

where $\mathbf{Q}_{1}=\mathbf{I}_{N_{p}} \otimes\left(\begin{array}{cc}2000 & 0 \\ 0 & 10000\end{array}\right), \mathbf{R}_{1}=5 \times 10^{5} \times \mathbf{I}_{N_{c}}$, and $\boldsymbol{\eta}_{\mathrm{dyn}, \mathrm{ref}}$ is the reference of $\boldsymbol{\eta}_{\mathrm{dyn}}, i=0,1,2, \ldots, N_{p}$.

Finally, by solving problem (42) with SDNN, obtain the control variable $\delta_{f}$ at the current moment and send it to the vehicle model.

The detailed flowchart of the proposed ACC system is presented in Figure 9.

\section{Simulations and Analyses}

To verify the capability of the proposed hybrid MPC algorithm for ACC, simulations have been carried out in Simulink. Its performance has been compared with an ACC system based on the Switch controller and an ACC system based on the Incremental PID controller under various working conditions. More precisely, the following mode is tested on straight roads in the following condition and gostop condition; cruising mode is tested on a straight road in the cruising condition; finally, the following mode is tested on a curved road in the curved-road condition to verify that the proposed ACC system has the ability to work on the curved roads. Parameters of the proposed ACC system, the vehicle, and the motor are listed in Tables 7-9, respectively.

4.1. Switch Controller in the Simulation. A Switch controller [33] has been used in the simulation for comparative analysis, which is divided into six working modes; the acceleration range of these six modes is listed in Table 10.

(1) Cruising mode:

$$
a_{\exp }= \begin{cases}2, & v_{1}<v_{c}^{d}-1, d \geq 50 \mathrm{~m}, \\ v_{c}^{d}-v_{1}, & v_{c}-1 \leq v_{1}<v_{c}^{d}+1, d \geq 50 \mathrm{~m}, \\ -2, & v_{1} \geq v_{c}^{d}+1, d \geq 50 \mathrm{~m} .\end{cases}
$$

(2) Approaching mode:

$$
a_{\exp }=-\frac{v_{r}^{2}}{2\left(d-d_{\mathrm{des}}\right)}, \quad 3+d_{\mathrm{des}} \leq d<50 \mathrm{~m}, v_{r}<-0.2 \mathrm{~km} / \mathrm{h} .
$$

(3) Accelerating mode:

$$
a_{\exp }=\left(\frac{1}{v_{1}}+1\right) v_{r}, \quad d_{\mathrm{des}}+3 \leq d<50 \mathrm{~m}, v_{r}>0 .
$$

(4) Following mode:

$$
a_{\exp }=\frac{1}{v_{1}}\left[\left(d-d_{\mathrm{des}}\right)+11 v_{r}\right], \quad d_{\mathrm{des}}-3 \leq d<d_{\mathrm{des}}+3 .
$$

(5) Decelerating mode:

$$
a_{\exp }=\frac{v_{r}^{2}}{\left(d-d_{\mathrm{des}}\right)}, \quad d_{\mathrm{safe}} \leq d<d_{\mathrm{des}}-3 .
$$

(6) Collision avoidance mode:

$$
a_{\exp }=-8 \mathrm{~m} / \mathrm{s}^{2}, \quad R<d_{\text {safe }} .
$$

4.2. Simulation in Four Conditions. The proposed ACC system is modelled in Simulink, and four simulations have been carried out in the following condition, go-stop condition, cruising condition for the straight roads, and curved-road condition for the curved road to test its performances.

4.2.1. Following Condition. Firstly, in the following condition, the MPC controller based on Quadprog, the MPC controller based on SDNN, the Switch controller, and the Incremental PID controller have been simulated. There are no build-in braking strategies in the Switch controller and the Incremental PID controller; for comparison, they are equipped with the braking strategy this paper proposed. $K_{P}$, $K_{I}$, and $K_{D}$ in the Incremental PID controller are set to 5000, 


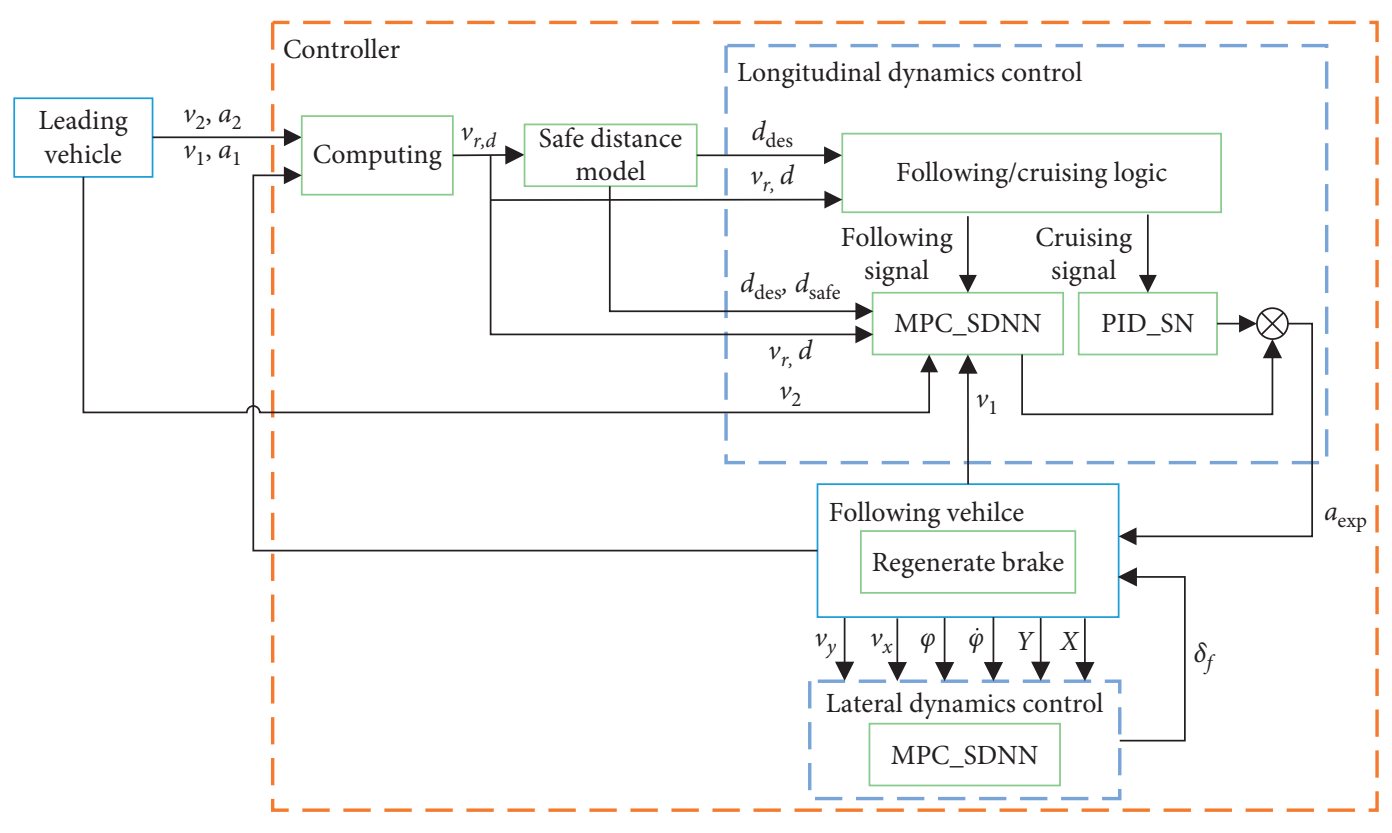

FIgURE 9: Flowchart of the proposed ACC system.

TABLE 7: Values of the parameters in the vehicle longitudinal dynamics and longitudinal dynamics controller design.

\begin{tabular}{lc}
\hline Parameter & Value \\
\hline$w_{\text {du }}$ & 100 \\
$T_{s} / \mathrm{s}$ & 0.001 \\
$\Delta d_{\text {sw }} / m$ & 6 \\
$\xi$ & 1 \\
$\varepsilon_{Q P}$ & 0.01 \\
$\varepsilon$ & 10 \\
$\Delta d_{\max }+d_{\text {des }}$ & 50 \\
$v_{r \min } /(\mathrm{km} / \mathrm{h})$ & -15 \\
$u_{\min } /\left(\mathrm{m} / \mathrm{s}^{2}\right)$ & -5 \\
$\Delta u_{\min } /\left(\mathrm{m} / \mathrm{s}^{2}\right)$ & 0.005 \\
$v_{c}^{d} /(\mathrm{km} / \mathrm{h})$ & 90 \\
$\mathbf{w}_{y}$ & Diag \\
$i n i t i a l ~ s O C$ & $0000,500,0,5)$ \\
$v_{r, \mathrm{sw}} /(\mathrm{km} / \mathrm{h})$ & 0.5 \\
$T_{\text {step }} / \mathrm{s}$ & 15 \\
$\rho$ & 0.5 \\
$\Delta d_{\max } / \mathrm{m}$ & 10 \\
$\Delta d_{\min } / \mathrm{m}$ & 10 \\
$v_{r \max } /(\mathrm{km} / \mathrm{h})$ & 3.6 \\
$u_{\max } /\left(\mathrm{m} / \mathrm{s}^{2}\right)$ & 15 \\
$\Delta u_{\max } /\left(\mathrm{m} / \mathrm{s}^{2}\right)$ & 3 \\
\hline
\end{tabular}

0 , and 0 , respectively. Figure 10 shows the speed profiles of the LV and the FV of the four controllers mentioned above, while Figure 11 shows three distances in the proposed ACC system. The tracking errors of speed and distance are shown in Figures 12 and 13, respectively.

Figure 12 demonstrates that the speed error of the MPC controller based on SDNN is nearly the same as the MPC controller based on Quadprog, while the speed error of the Incremental PID controller is the smallest. On the other hand, Figure 13 demonstrates that the distance error of the MPC controller based on SDNN is smaller than the MPC
TABLE 8: Values of the parameters of the vehicle model.

\begin{tabular}{lc}
\hline Parameter & Value \\
\hline$m / \mathrm{kg}$ & 1270.000 \\
$f$ & 0.0196 \\
$R / \mathrm{m}$ & 0.325 \\
$C_{D}$ & 0.300 \\
$A / \mathrm{m}^{2}$ & 2.200 \\
$\eta$ & 0.900 \\
$i_{t}$ & 1.400 \\
\hline
\end{tabular}

TABle 9: Values of the parameters of PMSM.

\begin{tabular}{lc}
\hline Parameter & Value \\
\hline Rated power/kW & 50 \\
Rated speed/rpm & 4000 \\
Rated current/A & 365 \\
Number of phases & 3 \\
q-axis inductance/mH & 0.357 \\
Stator resistor/m $\Omega$ & 6.66 \\
Rated voltage/V & 350 \\
Rated torque/Nm & 120 \\
Number of pole-pairs & 4 \\
d-axis inductance/mH & 0.135 \\
Permanent flux $/ \mathrm{Wb}$ & 0.062 \\
Rotational inertia/ $/ \mathrm{kgm}^{2}$ & 0.044 \\
\hline
\end{tabular}

TABLE 10: Values of the acceleration range in six modes.

\begin{tabular}{lc}
\hline Mode & Acceleration range $\left(\mathrm{m} / \mathrm{s}^{2}\right)$ \\
\hline Cruising mode & $(-2,2)$ \\
Approach mode & $(-3,0)$ \\
Accelerating mode & $(0,3)$ \\
Following mode & $(-1,1)$ \\
Decelerating mode & $(-6,0)$ \\
Collision avoidance mode & -8
\end{tabular}




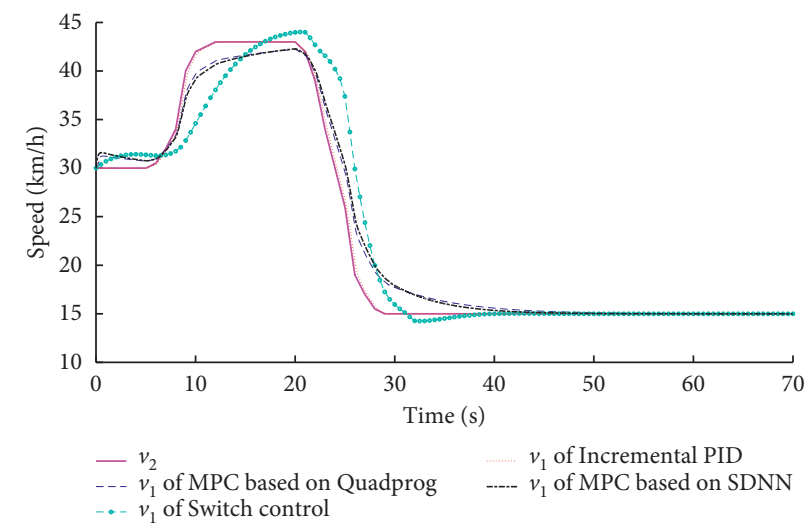

Figure 10: Speeds of the LV and the FV of four controllers.
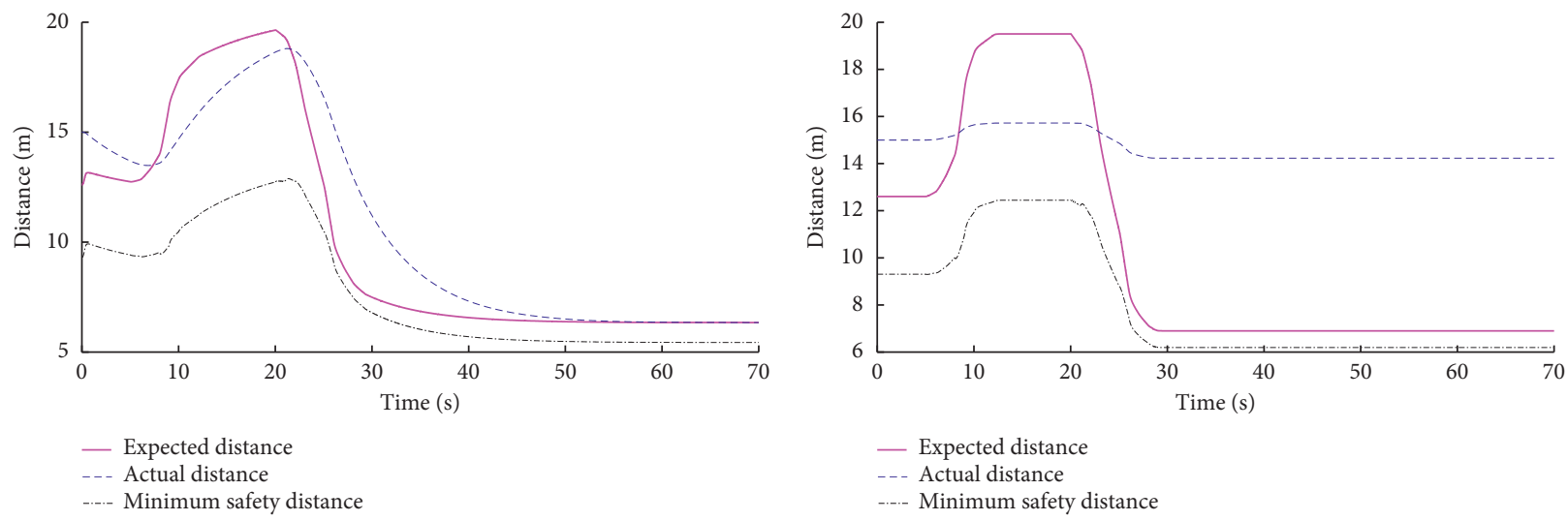

(a)

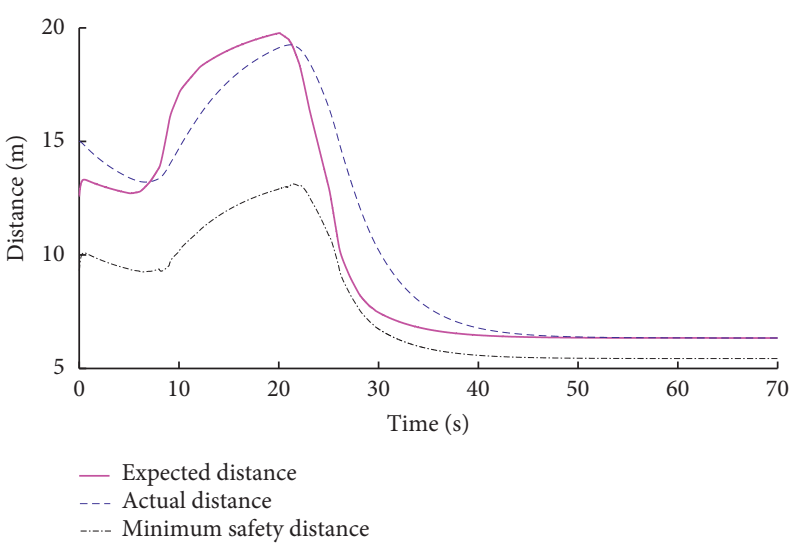

(c)

(d)

FIgURE 11: Distances of the LV and the FV of four controllers. (a) The MPC controller based on Quadprog. (b) The Incremental PID controller. (c) The Switch controller. (d) The MPC controller based on SDNN.

controller based on Quadprog, and the distance error of the Incremental PID controller is the biggest of all controllers in the test. To further evaluate the tracking performance, the absolute average errors and the root-mean-square of errors are listed in Table 11. In the following condition, the average distance error of the MPC controller based on SDNN is $29.98 \%$ lower than that of the MPC controller based on
Quadprog while the average speed errors are essentially equal.

Total braking energy and regenerative braking energy of four controllers are shown in Figures 14 and 15, respectively. Table 12 demonstrates that the energy recovery efficiency of the SDNN based MPC controller is the highest among the four controllers. In addition, the 


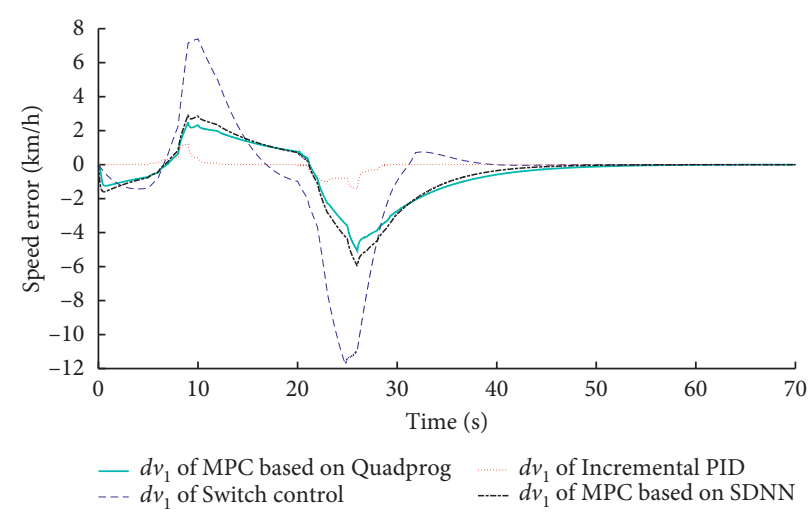

Figure 12: Speed errors of the LV and the FV of four controllers.

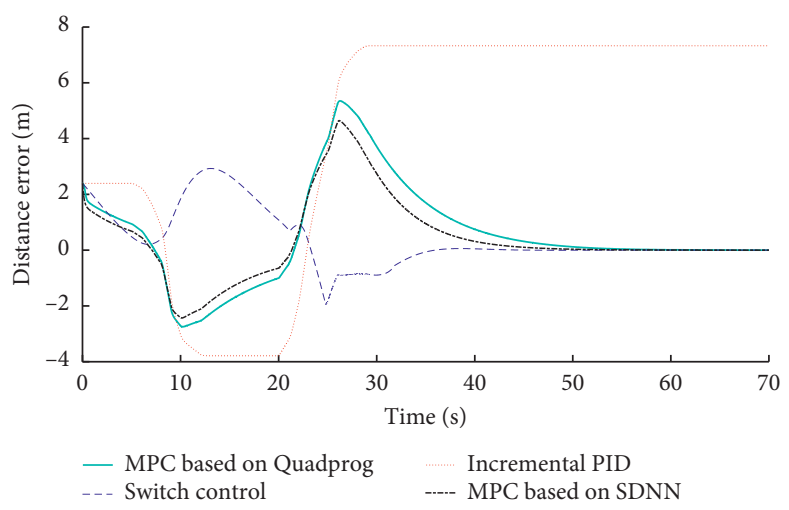

FIgURE 13: Distance errors of the LV and the FV of four controllers.

computation of QP by SDNN is $23.81 \%$ faster than the Quadprog function as shown in Table 11. SDNN used to accelerate the computation of the QP problem of MPC is confirmed to be effective.

Though the speed error of the Incremental PID controller is the smallest, considering all the factors including energy economy and computation efficiency, the MPC controller based on SDNN is the best controller in the following condition, which has achieved better tracking performance than other controllers.

4.2.2. Go-Stop Condition. In the go-stop condition, the MPC controller based on Quadprog, the MPC controller based on SDNN, the Switch controller, and the Incremental PID controller have been simulated. For the same reason, the Switch controller and the Incremental PID controller are equipped with the braking strategy this paper proposed. $K_{P}$, $K_{I}$, and $K_{D}$ in the Incremental PID controller are set to 5000, 0 , and 0 , respectively. Figure 16 shows the speeds of the LV and the FV of four controllers mentioned above, while Figure 17 shows three distances in the proposed ACC system. The tracking errors of speed and distance are shown in Figures 18 and 19, respectively. Figure 18 demonstrates that the speed error of the MPC controller based on SDNN is nearly the same as the MPC controller based on Quadprog, while the speed error of the Incremental PID controller is the smallest. On the other hand, Figure 19 demonstrates that the distance error of the MPC controller based on SDNN is smaller than the MPC controller based on Quadprog, and the distance error of the Incremental PID controller is the biggest of all controllers in the test. The absolute average errors and the root-mean-square of errors are listed in Table 13. In the go-stop condition, the average distance error of the MPC controller based on SDNN is 55.37\% lower than that of the MPC controller based on Quadprog while the average speed error of the MPC controller based on SDNN is $64.16 \%$ lower than that of the MPC controller based on Quadprog.

The total braking energy and regenerative braking energy of four controllers are shown in Figures 20 and 21, respectively. Table 14 demonstrates that the energy recovery efficiency of the SDNN based MPC controller is highest among the four controllers the same as in the following condition. In addition, the computation of QP by SDNN is 23.39\% faster than the Quadprog function as shown in Table 13. SDNN used to accelerate the computation of the QP problem of MPC takes effects.

Despite the fact that the speed error of the Incremental PID controller is the smallest, considering all the factors, the MPC controller based on SDNN is the best controller in the go-stop condition. Similar to the following condition, the MPC controller based on SDNN has achieved better tracking performance than other controllers. 
TABLE 11: Performance in the following condition.

\begin{tabular}{lcccc}
\hline & \multicolumn{2}{c}{ Following condition } & & \\
& MPC_Quadprog & MPC_SDNN & Switch & Incremental PID \\
\hline Average speed error (absolute value) $(\mathrm{km} / \mathrm{h})$ & 0.4451 & 0.4452 & 0.4452 & 0.0395 \\
Root-mean-square of speed error & 1.5053 & 1.7238 & 3.1719 & 0.3022 \\
Average distance error (absolute value) $(\mathrm{m})$ & 0.5536 & 0.3876 & 0.3781 & 4.2940 \\
Root-mean-square of distance error & 1.8286 & 1.4706 & 1.0700 & 6.0736 \\
Simulation time (s) & 4101.5622 & 3125.1653 & 2960.1980 & 2741.1237 \\
\hline
\end{tabular}

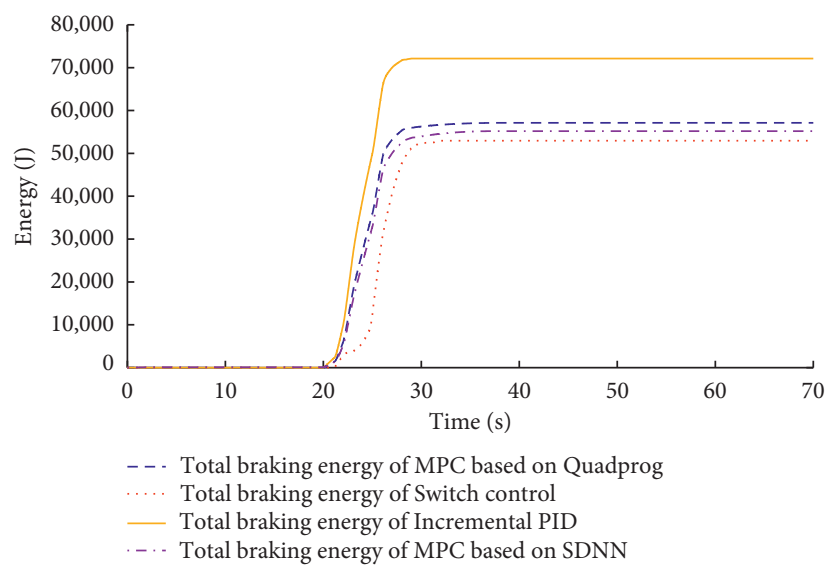

Figure 14: Total braking energy of four controllers.

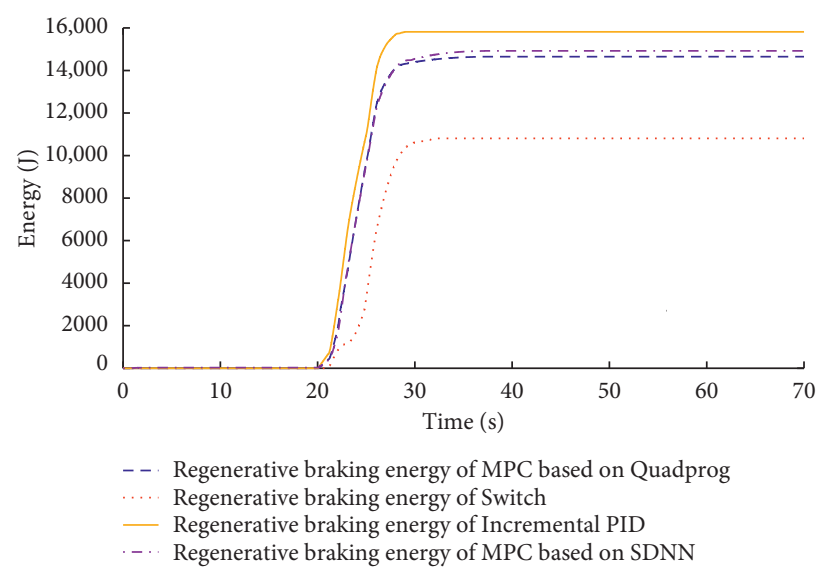

FIGURE 15: Regenerative braking energy of four controllers.

TABLE 12: Performance of energy recovery in braking in the following condition.

\begin{tabular}{lcccc}
\hline & \multicolumn{2}{c}{ Following condition } & & \\
& MPC_Quadprog & Incremental PID & Switch & MPC_SDNN \\
\hline Total braking energy $/\left(10^{4} \mathrm{~J}\right)$ & 5.7126 & 7.2134 & 5.2961 & 5.5202 \\
Regenerative braking energy $/\left(10^{4} \mathrm{~J}\right)$ & 1.4653 & 1.5816 & 1.0810 & 1.4921 \\
Energy recovery efficiency $(\%)$ & 25.6 & 21.9 & 20.4 & 27.0 \\
\hline
\end{tabular}

4.2.3. Cruising Condition. In the cruising condition, the PID controller based on SN, the Switch controller, and the Incremental PID controller have been simulated. $K_{P}, K_{I}$, and $K_{D}$ in the Incremental PID controller are set to 800,0 , and 0 , respectively. Figure 22 shows the LV and the FV of the three controllers mentioned above. The tracking errors of speed and distance are shown in Figure 23.

As shown in Table 15, the average speed error of the PID controller based on SN is $32.89 \%$ lower than that of the Switch controller and $7.93 \%$ lower than that of the 


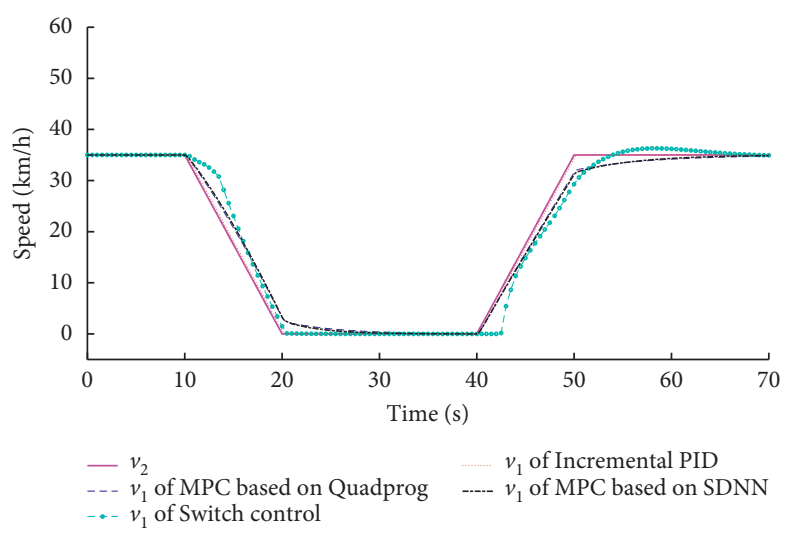

FIgURE 16: Speeds of the LV and the FV of four controllers.
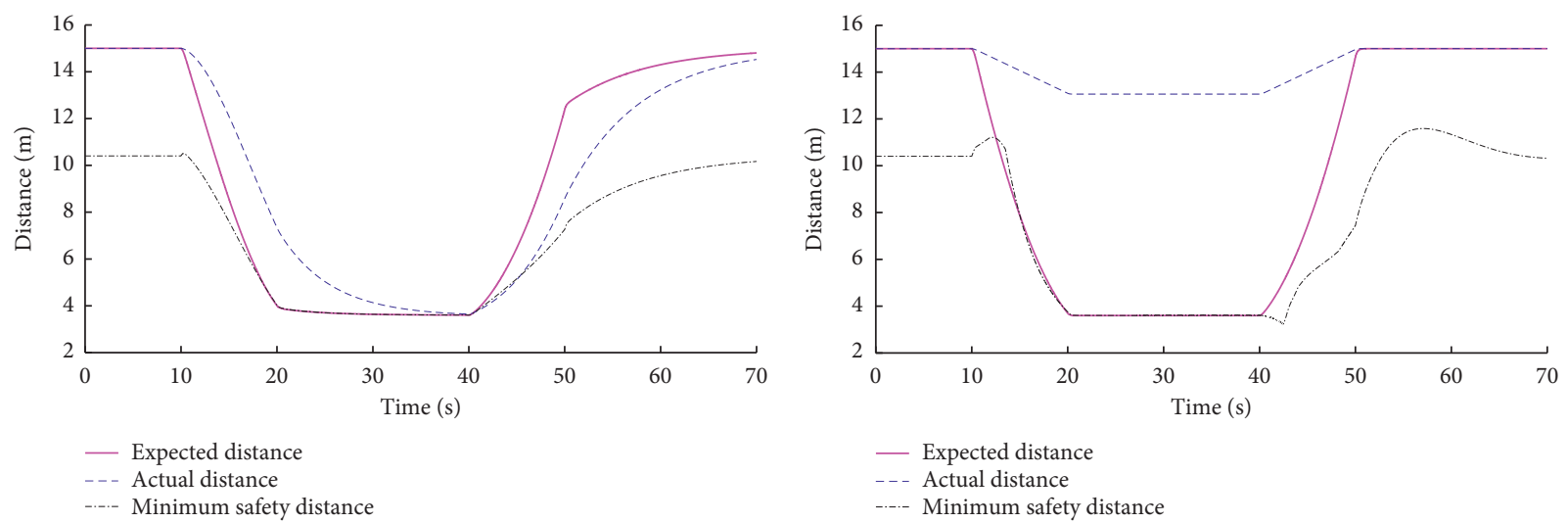

(a)

(b)
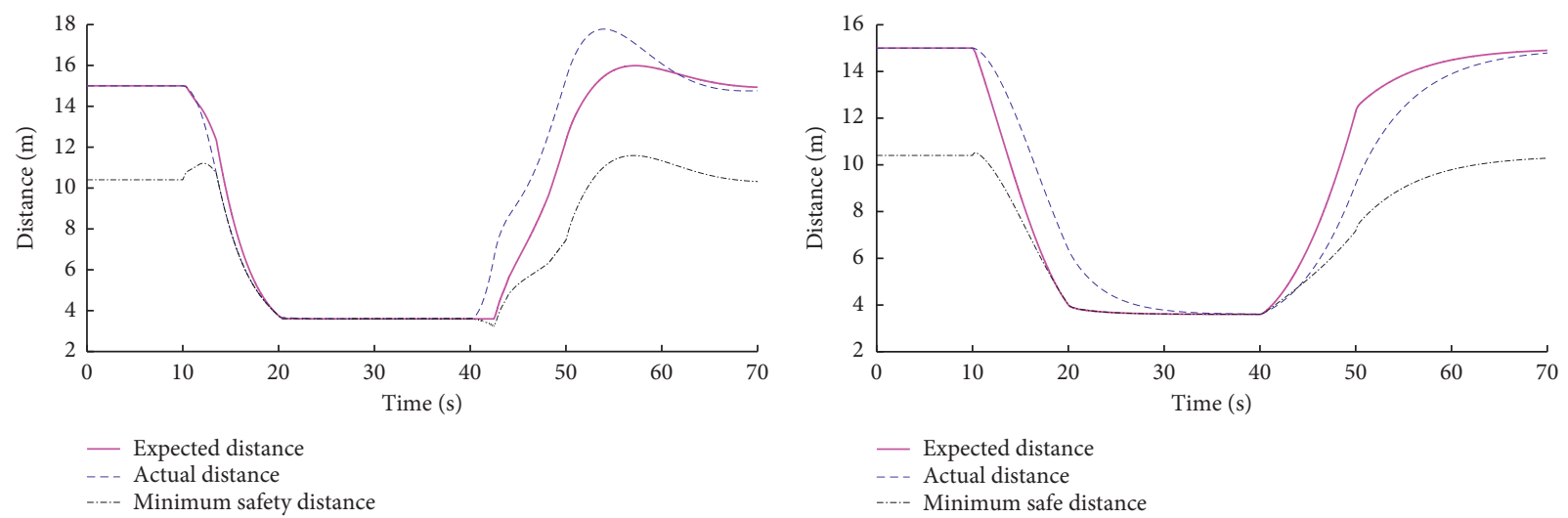

(c)

(d)

Figure 17: Distances of the LV and the FV of four controllers. (a) The MPC controller based on Quadprog. (b) The Incremental PID controller. (c) The Switch controller. (d) The MPC controller based on SDNN.

Incremental PID controller while the speed error (RMS $\left(v_{r}\right)$ ) of the PID controller based on SN is $18.15 \%$ lower than that of the Switch controller. The PID controller based on SN has achieved better tracking performance than other controllers.
4.2.4. Curved-Road Condition. The curved-road condition is set to verify that the proposed ACC system has the ability to work on the curved roads [34]. In this condition, the Switch controller and the Incremental PID controller cannot work 


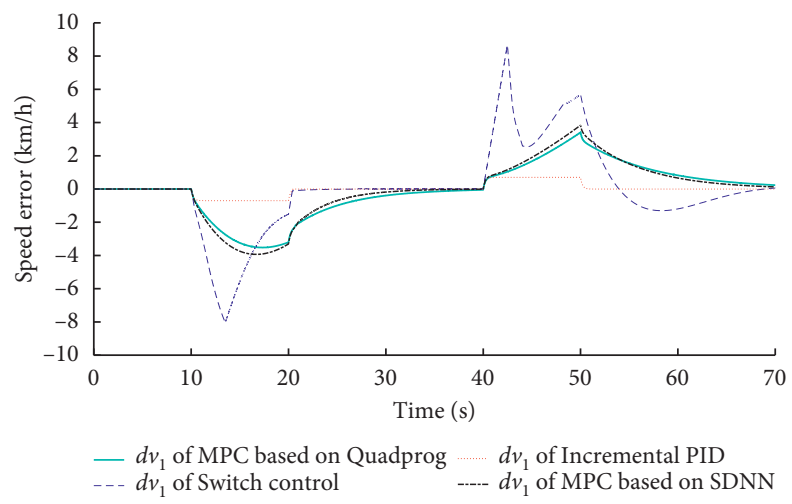

FIgURE 18: Speed errors of the LV and the FV of four controllers.

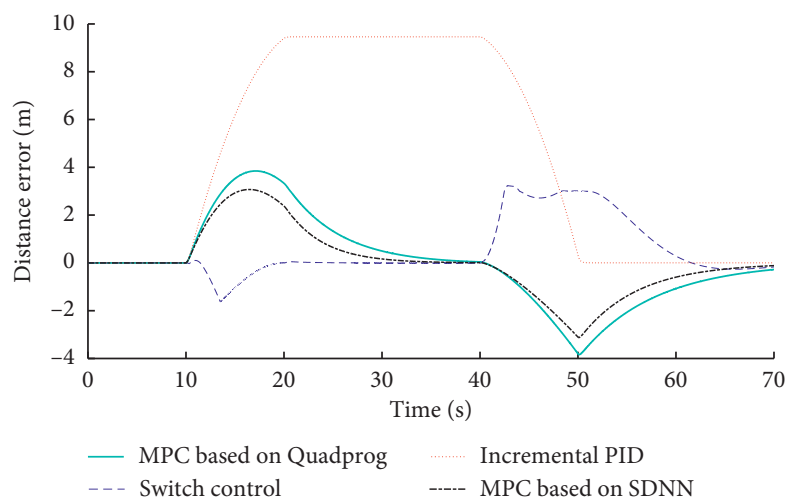

FIgURE 19: Distance errors of the LV and the FV of four controllers.

TABle 13: Performance in the go-stop condition.

\begin{tabular}{lcccc}
\hline & Go-stop condition & & & \\
& MPC_Quadprog & MPC_SDNN & Switch & Incremental PID \\
\hline Average speed error (absolute value) $(\mathrm{km} / \mathrm{h})$ & 0.0242 & 0.0108 & 0.0121 & 0.0003 \\
Root-mean-square of speed error & 1.5797 & 1.7140 & 2.5491 & 0.3703 \\
Average distance error (absolute value) $(\mathrm{m})$ & 0.0399 & 0.0143 & 0.5207 & 4.3696 \\
Root-mean-square of distance error & 1.7811 & 1.3550 & 1.2963 & 6.1211 \\
Simulation time (s) & 4112.4813 & 3150.5771 & 2971.6121 & 2753.1442 \\
\hline
\end{tabular}

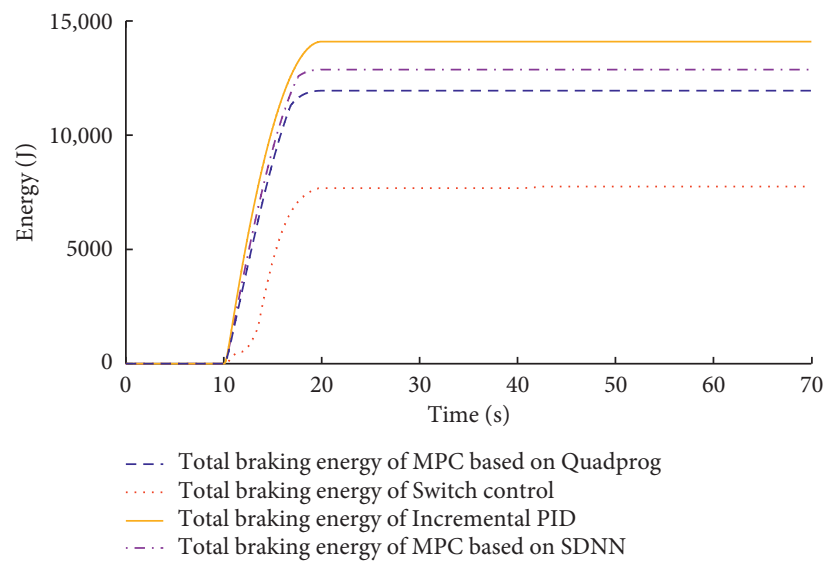

Figure 20: Total braking energy of four controllers. 


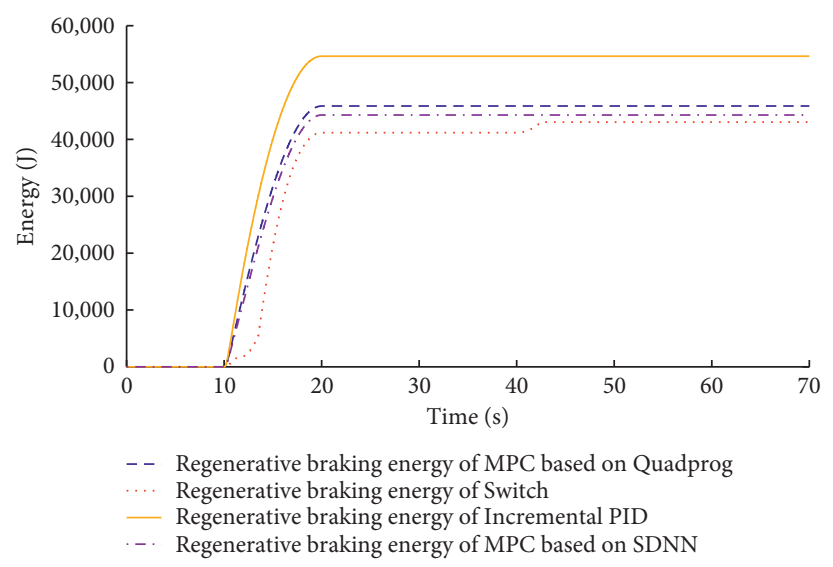

FIGURE 21: Regenerative braking energy of four controllers.

TABle 14: Performance of energy recovery in braking in the go-stop condition.

\begin{tabular}{lcccc}
\hline & \multicolumn{2}{c}{ Go-stop condition } & & \\
& MPC_Quadprog & Incremental PID & Switch & MPC_SDNN \\
\hline Total braking energy $\left(10^{4} \mathrm{~J}\right)$ & 4.4300 & 5.4641 & 4.3056 & 4.5876 \\
Regenerative braking energy $\left(10^{4} \mathrm{~J}\right)$ & 1.1948 & 1.4096 & 0.7755 & 1.2869 \\
Energy recovery efficiency $(\%)$ & 27.0 & 25.8 & 18.0 & 28.1 \\
\hline
\end{tabular}

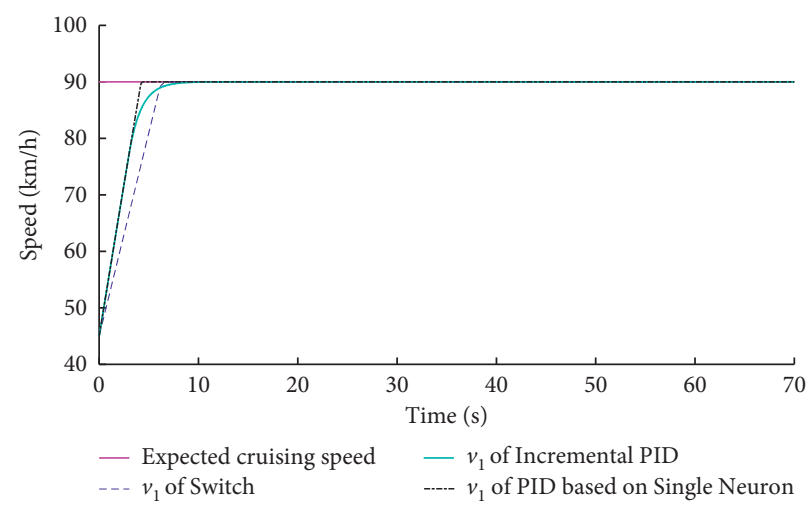

FIgURE 22: Speeds of the LV and the FV of three controllers.

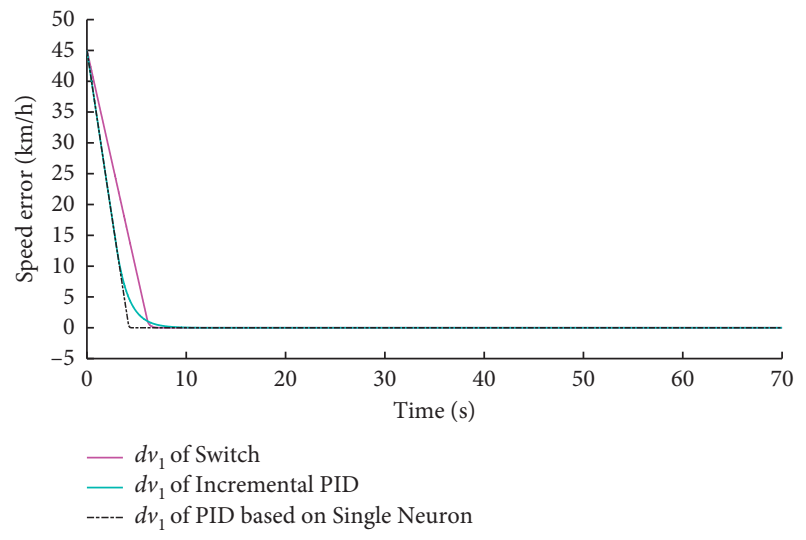

FIGURE 23: Speed errors of the LV and the FV of three controllers. on the curved road, so only the MPC controller based on Quadprog and the MPC controller based on SDNN are tested. The reference trajectory is set as follows:

$$
\begin{aligned}
& \mathbf{Y}_{\text {ref }}(X)=\frac{d_{\mathbf{y}_{1}}}{2}\left(1+\tanh \left(z_{1}\right)\right)-\frac{d_{\mathbf{y}_{2}}}{2}\left(1+\tanh \left(z_{2}\right)\right), \\
& \arctan \left(d_{\mathbf{y}_{1}}\left(\frac{1}{\cosh \left(z_{1}\right)}\right)^{2}\left(\frac{1.2}{d_{\mathbf{x}_{1}}}\right)-d_{\mathbf{y}_{2}}\left(\frac{1}{\cosh \left(z_{2}\right)}\right)^{2}\left(\frac{1.2}{d_{\mathbf{x}_{2}}}\right)\right),
\end{aligned}
$$

where $z_{1}=(2.4 / 25)(X-27.19)-1.2 ; z_{2}=(2.4 / 21.95)(X-$ $56.46)-1.2 ; d_{\mathbf{x}_{1}}=25 ; d_{\mathbf{x}_{2}}=21.95 ; d_{\mathbf{y}_{1}}=4.05$; and $d_{\mathbf{y}_{2}}=5.7$.

Figure 24 shows the trajectories on the curved road while Figure 25 shows the lateral motion errors of the two controllers mentioned above. It is apparent that the lateral 
TABle 15: Performance in the cruising condition.

\begin{tabular}{lccc}
\hline & Cruising condition & & \\
& PID based on SN & Switch & Incremental PID \\
\hline Average speed error (absolute value) $(\mathrm{km} / \mathrm{h})$ & 1.3503 & 2.0122 & 1.4666 \\
Root-mean-square of speed error & 6.3549 & 7.7642 & 6.3947 \\
\hline
\end{tabular}

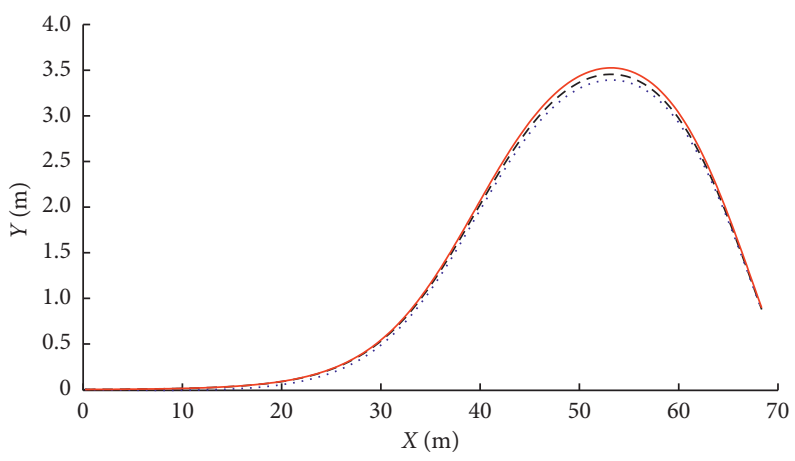

Reference trajectory

Trajectory of MPC based on Quadprog

- - - Trajectory of MPC based on SDNN

Figure 24: Trajectories on the curved road.

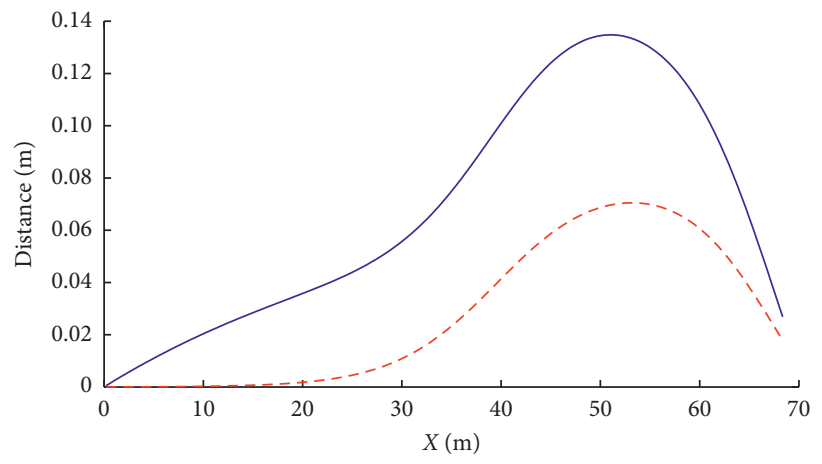

— Lateral motion of MPC based on Quadprog from $Y_{\text {ref }}$

- - - Lateral motion of MPC based on SDNN from $Y_{\text {ref }}$

FIgURE 25: Lateral motion errors of two controllers.

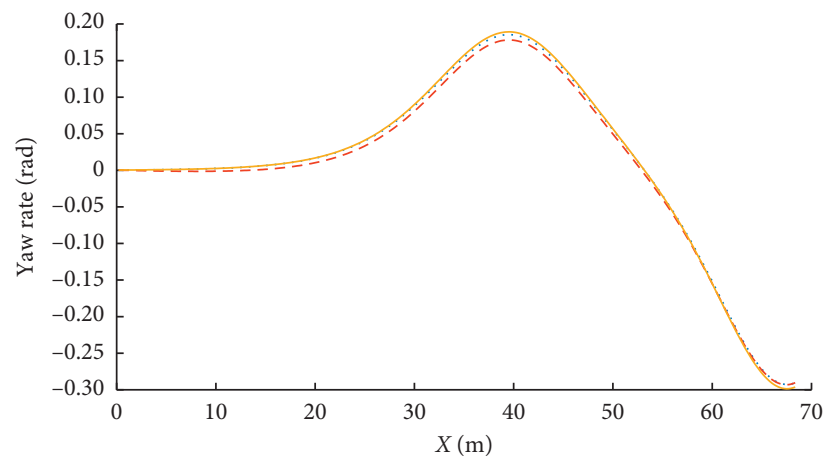

$-\varphi_{\text {ref }}$

- - $\varphi$ of MPC based on Quadprog $\varphi$ of MPC based on SDNN

Figure 26: Yaw angles of two controllers. 


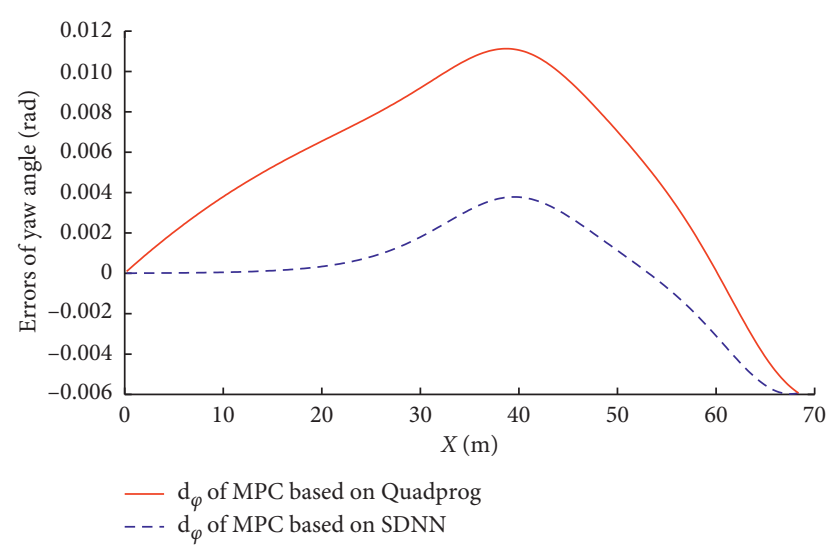

FiguRE 27: Errors of yaw angle of two controllers.

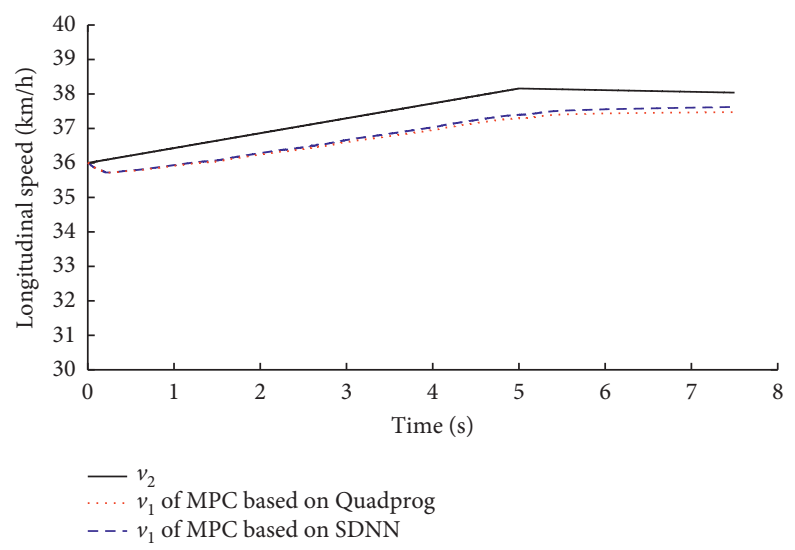

Figure 28: Longitudinal speeds of the LV and the FV of two controllers.

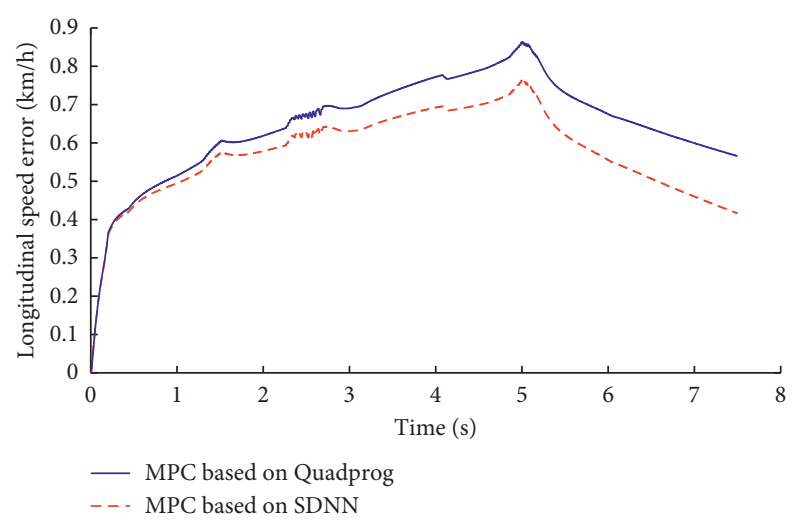

FIgURE 29: Longitudinal speed errors of the LV and the FV of two controllers.

motion error of the MPC controller based on SDNN is smaller than the MPC controller based on Quadprog.

Yaw angles and errors of yaw angle are presented in Figures 26 and 27, respectively.

The error of yaw angle of the MPC controller based on SDNN is also smaller than the MPC controller based on Quadprog, so the longitudinal speeds and errors of longitudinal speed are presented in Figures 28 and 29. The absolute average errors and the root-mean-square of errors are listed in Table 16. In the curved-road condition, the average error of lateral motion of the MPC controller based on SDNN is $59.64 \%$ lower than that of the MPC controller based on Quadprog while the average error of yaw angle of the MPC controller based on SDNN is $70.49 \%$ lower than that of the MPC controller based on Quadprog.

Total braking energy and regenerative braking energy of two controllers are shown in Figure 30. Table 17 demonstrates that the energy recovery efficiency of the SDNN based 
TABLE 16: Values of the parameters in the lateral dynamics controller design.

\begin{tabular}{lc}
\hline Parameter & Value \\
\hline$I_{z} / \mathrm{kg} \cdot \mathrm{m}^{2}$ & 4175.000 \\
$l_{r} / \mathrm{m}$ & 1.468 \\
$C_{\mathrm{cr}} /(\mathrm{N} / \mathrm{rad})$ & 62700.000 \\
$C_{\mathrm{lr}} /(\mathrm{N} / \mathrm{rad})$ & 62700.000 \\
$\Delta u_{\max }^{\prime} / \mathrm{rad}$ & 0.0148 \\
$u_{\min }^{\prime} / \mathrm{rad}$ & -0.1744 \\
$Y_{\max } / \mathrm{m}$ & -1 \\
$\varphi_{\min } / \mathrm{rad}$ & 5 \\
$l_{f} / \mathrm{m}$ & 1.232 \\
$C_{\mathrm{cf}} /(\mathrm{N} / \mathrm{rad})$ & 66900.000 \\
$C_{\mathrm{lf}} /(\mathrm{N} / \mathrm{rad})$ & 66900.000 \\
$\Delta u_{\max }^{\prime} / \mathrm{rad}$ & 0.0148 \\
$u_{\max }^{\prime} / \mathrm{rad}$ & 0.1744 \\
$Y_{\max } / \mathrm{m}$ & 5 \\
$\varphi_{\max } / \mathrm{rad}$ & 0.21 \\
\hline
\end{tabular}

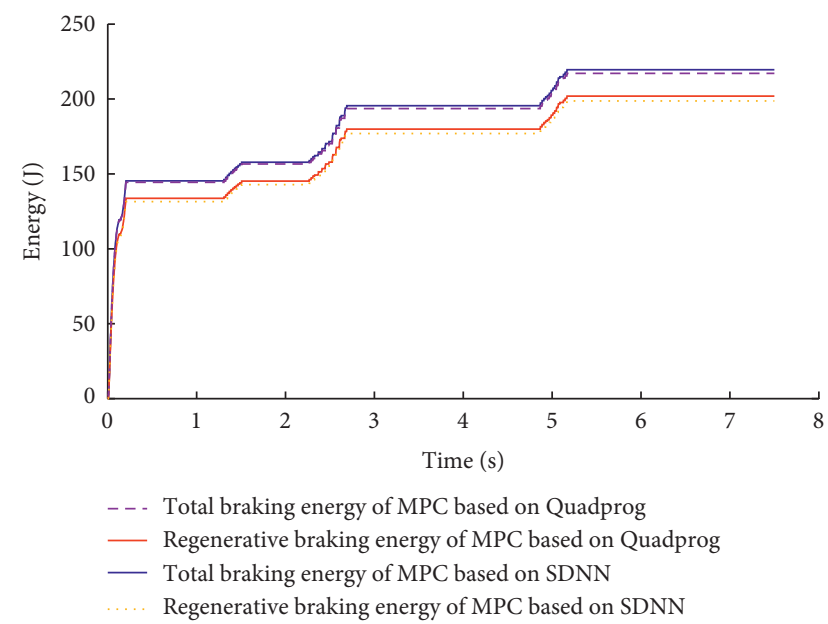

FIgURE 30: Braking energies of two controllers.

TABLE 17: Performance of energy recovery in braking in the curvedroad condition.

\begin{tabular}{lcc}
\hline \multicolumn{3}{c}{ Curved-road condition } \\
& MPC_Quadprog & MPC_SDNN \\
\hline Total braking energy (J) & 226.8117 & 229.3997 \\
Regenerative braking energy (J) & 207.6067 & 211.0447 \\
Energy recovery efficiency (\%) & 91.5 & 92.0 \\
\hline
\end{tabular}

MPC controller is higher. In addition, the computation of QP by SDNN is $16.69 \%$ faster than the Quadprog function as shown in Table 18 which shows that the SDNN is effective as expected. In conclusion, the proposed ACC system can work for the curved road.
TABle 18: Performance in the curved-road condition.

\begin{tabular}{lcc}
\hline \multicolumn{3}{c}{ Curved-road condition } \\
& MPC_Quadprog & MPC_SDNN \\
\hline $\begin{array}{l}\text { Average longitudinal speed } \\
\text { error (absolute value) }(\mathrm{km} / \mathrm{h})\end{array}$ & 0.6477 & 0.5728 \\
$\begin{array}{l}\text { Root-mean-square of } \\
\text { longitudinal speed error } \\
\text { Average error of lateral motion } \\
\text { (absolute value) (m) }\end{array}$ & 0.6605 & 0.5841 \\
$\begin{array}{l}\text { Root-mean-square of error of } \\
\text { lateral motion }\end{array}$ & 0.0674 & 0.0272 \\
$\begin{array}{l}\text { Average error of yaw angle } \\
\text { (absolute value) (rad) }\end{array}$ & 0.0061 & 0.0382 \\
$\begin{array}{l}\text { Root-mean-square of error of } \\
\text { yaw angle }\end{array}$ & 0.0069 & 0.0018 \\
Simulation time (s) & 365.2265 & 304.2355 \\
\hline
\end{tabular}

\section{Conclusion}

This paper presented a hybrid MPC controller based on a simplified dual neural network (SDNN) and PID based on single neuron (SN) for adaptive cruise control of autonomous electric vehicles to incorporate the performances of tracking, comfort, safety, and energy economy by considering spatiotemporal constraints between the leading vehicle and the following vehicle. The driving modes were divided into following and cruising, and the MPC algorithm based on SDNN and the PID controller based on SN were applied to the following mode and the cruising mode, respectively. Moreover, lateral dynamics control was taken into consideration which is often ignored by conventional ACC systems, so the proposed ACC system could work on the curved road. In addition, the braking strategy was also verified to be effective in the simulations.

Simulation results have shown that the average distance error of the MPC controller in the following condition based on SDNN was $29.98 \%$ lower than that of the MPC controller based on Quadprog while the average speed errors were essentially equal. In addition, the computation of QP by SDNN was $24.01 \%$ faster than the Quadprog function. And the MPC controller based on SDNN outperformed other controllers in the go-stop condition, the average distance error of the MPC controller based on SDNN was $55.37 \%$ lower than that of the MPC controller based on Quadprog while the average speed error of the MPC controller based on SDNN was $64.16 \%$ lower than that of the MPC controller based on Quadprog. Furthermore, the computation of QP by SDNN was 23.39\% faster than Quadprog function. Compared with the conventional numerical solution as Quadprog function, the calculation in SDNN did not involve matrix inversion, decomposition, and other operations. So, the computational complexity to solve QP of SDNN was lower. 
In the cruising condition, the PID controller based on SN achieved better tracking performance than other controllers. The average speed error of the PID controller based on SN was $32.89 \%$ lower than that of the Switch controller and 7.93\% lower than that of the Incremental PID controller while the speed error (RMS $\left(v_{r}\right)$ ) of the PID controller based on SN was $18.15 \%$ lower than that of the Switch controller. All of this was due to the strong adaptive capability of the PID controller based on SN. Additionally, the curved-road condition verified that the proposed ACC system had the ability to work on the curved roads which was as expected.

In general, results validated that the proposed strategy is effective for adaptive cruise control of autonomous electric vehicles.

\section{Data Availability}

The data used to support the findings of this study are available from the corresponding author upon request.

\section{Conflicts of Interest}

The authors declare that there are no conflicts of interest regarding the publication of this article.

\section{Acknowledgments}

This research was supported in part by the Key-Area Research and Development Program of Guangdong Province under Grant 2020B090921003 and in part by the Key-Area Research and Development Program of Guangdong Province under Grant 2020B0909030005.

\section{References}

[1] K. Bengler, K. Dietmayer, B. Farber, M. Maurer, C. Stiller, and H. Winner, "Three decades of driver assistance systems: review and future perspectives," IEEE Intelligent Transportation Systems Magazine, vol. 6, no. 4, pp. 6-22, 2014.

[2] Z. Bareket, P. S. Fancher, H. Peng, and K. Lee, C. A. Assaf, Methodology for assessing adaptive cruise control behavior," IEEE Transactions on Intelligent Transportation Systems, vol. 4, no. 3, pp. 123-131, 2003.

[3] B. Ganji, A. Z. Kouzani, S. Y. Khoo, and M. Shams-Zahraei, "Adaptive cruise control of a HEV using sliding mode control," Expert Systems with Applications, vol. 41, no. 2, pp. 607-615, 2014.

[4] V. Milanés, S. E. Shladover, J. Spring et al., "Cooperative adaptive cruise control in real traffic situations," IEEE Transactions on Intelligent Transportation Systems, vol. 15, no. 1, pp. 296-305, 2013.

[5] M. Bichi, G. Ripaccioli, S. Di Cairano et al., "Stochastic model predictive control with driver behavior learning for improved powertrain control," in Proceedings of the 49th IEEE Conference on Decision and Control (CDC), pp. 6077-6082, Atlanta, GA, USA, December 2010.

[6] P. Shakouri and A. Ordys, "Nonlinear model predictive control approach in design of adaptive cruise control with automated switching to cruise control," Control Engineering Practice, vol. 26, pp. 160-177, 2014.

[7] J.-J. Martinez and C. Canudas-de-Wit, "A safe longitudinal control for adaptive cruise control and stop-and-go scenarios," IEEE Transactions on Control Systems Technology, vol. 15, no. 2, pp. 246-258, 2007.

[8] S. G. Kim, M. Tomizuka, and K. H. Cheng, "Smooth motion control of the adaptive cruise control system by a virtual lead vehicle," International Journal of Automotive Technology, vol. 13, no. 1, p. 77, 2012.

[9] C. U. Mba and C. Novara, "Optimal strategies for adaptive cruise control," Cities, Green Technologies, and Intelligent Transport Systems, Springer, Cham, Switzerland, 2016.

[10] H. Kim and K. Yi, "Design of a model reference cruise control algorithm," SAE International Journal of Passenger CarsElectronic and Electrical Systems, vol. 5, pp. 440-449, 2012.

[11] S. Li, K. Li, R. Rajamani et al., "Model predictive multi-objective vehicular adaptive cruise control[J]," IEEE Transactions on Control Systems Technology, vol. 19, no. 3, pp. 556-566, 2010.

[12] D. Zhao and Z. Hu, "Supervised adaptive dynamic programming based adaptive cruise control," in Proceedings of the 2011 IEEE Symposium on Adaptive Dynamic Programming and Reinforcement Learning (ADPRL), pp. 318-323, IEEE, Paris, France, April 2011.

[13] Z. H. Hu and D. B. Zhao, "Supervised reinforcement learning for adaptive cruise control," in Proceedings of the 4th International Symposium on Computational Intelligence and Industrial Application, pp. 239-248, Hangzhou, China, October 2010.

[14] V. Milanés, J. Villagrá, J. Pérez et al., "Low-speed longitudinal controllers for mass-produced cars: a comparative study," IEEE Transactions on Industrial Electronics, vol. 59, no. 1, pp. 620-628, 2011.

[15] E. Kural and B. A. Güvenç, "Model predictive adaptive cruise control," in Proceedings of the 2010 IEEE International Conference on Systems, Man and Cybernetics, pp. 1455-1461, Istanbul, Turkey, October 2010.

[16] R. Kanjee, A. K. Bachoo, and J. Carroll, "Vision-based adaptive cruise control using pattern matching," in Proceedings of the 2013 6th Robotics and Mechatronics Conference (RobMech), pp. 93-98, Durban, South Africa, October 2013.

[17] M. Vajedi and N. L. Azad, "Ecological adaptive cruise controller for plug-in hybrid electric vehicles using nonlinear model predictive control," IEEE Transactions on Intelligent Transportation Systems, vol. 17, no. 1, pp. 113-122, 2015.

[18] C. Koffler and K. Rohde-Brandenburger, "On the calculation of fuel savings through lightweight design in automotive life cycle assessments," The International Journal of Life Cycle Assessment, vol. 15, no. 1, p. 128, 2010.

[19] K. Fukuo, A. Fujimura, M. Saito et al., "Development of the ultra-low-fuel-consumption hybrid car-insight," JSAE Review, vol. 22, no. 1, pp. 95-103, 2001.

[20] H. Zhang, J. Wang, and Y. Y. Wang, "Optimal dosing and sizing optimization for a ground-vehicle diesel-engine twocell selective catalytic reduction system," IEEE Transactions on Vehicular Technology, vol. 65, no. 6, pp. 4740-4751, 2015.

[21] X. Hu, N. Murgovski, L. M. Johannesson et al., "Optimal dimensioning and power management of a fuel cell/battery hybrid bus via convex programming," IEEE/ASME Transactions on Mechatronics, vol. 20, no. 1, pp. 457-468, 2014.

[22] H. Zhang and J. Wang, "Vehicle lateral dynamics control through AFS/DYC and robust gain-scheduling approach," IEEE Transactions on Vehicular Technology, vol. 65, no. 1, pp. 489-494, 2015.

[23] R.-H. Zhang, Z.-C. He, H.-W. Wang, F. You, and K.-N. Li, "Study on self-tuning tyre friction control for developing 
main-servo loop integrated chassis control system," IEEE Access, vol. 5, pp. 6649-6660, 2017.

[24] X. Tang, T. Jia, X. Hu et al., "Naturalistic data-driven predictive energy management for plug-in hybrid electric vehicles," IEEE Transactions on Transportation Electrification, 2020.

[25] H. Liu, Y. Lei, Y. Fu, and X. Li, "An optimal slip ratio-based revised regenerative braking control strategy of range-extended electric vehicle," Energies, vol. 13, no. 6, p. 1526, 2020.

[26] K. Tanaka, T. Hori, and H. O. Wang, "A multiple Lyapunov function approach to stabilization of fuzzy control systems," IEEE Transactions on Fuzzy Systems, vol. 11, no. 4, pp. 582$589,2003$.

[27] G. Feng, "A survey on analysis and design of model-based fuzzy control systems," IEEE Transactions on Fuzzy Systems, vol. 14, no. 5, pp. 676-697, 2006.

[28] T. Liu, J. Zheng, Y. Su et al., "A study on control strategy of regenerative braking in the hydraulic hybrid vehicle based on ECE regulations," Mathematical Problems in Engineering, vol. 2013, Article ID 208753, 2013.

[29] P. Shakouri, A. Ordys, and M. R. Askari, "Adaptive cruise control with stop\&go function using the state-dependent nonlinear model predictive control approach," ISA Transactions, vol. 51, no. 5, pp. 622-631, 2012.

[30] S. H. Hosseinnia, I. Tejado, V. Milanés, J. Villagra, and B. M. Vinagre, "Experimental application of hybrid fractionalorder adaptive cruise control at low speed," IEEE Transactions on Control Systems Technology, vol. 22, no. 6, pp. 2329-2336, 2014.

[31] S. Liu and J. Wang, "A simplified dual neural network for quadratic programming with its KWTA application," IEEE Transactions on Neural Networks, vol. 17, no. 6, pp. 1500-1510, 2006.

[32] W. Liu and Z. Li, "Comprehensive predictive control method for automated vehicles with delays," IEEE Access, vol. 7, pp. 81923-81933, 2019.

[33] S. Moon and K. Yi, "Human driving data-based design of a vehicle adaptive cruise control algorithm," Vehicle System Dynamics, vol. 46, no. 8, pp. 661-690, 2008.

[34] P. Falcone, H. Eric Tseng, F. Borrelli, J. Asgari, and D. Hrovat, "MPC-based yaw and lateral stabilisation via active front steering and braking," Vehicle System Dynamics, vol. 46, no. S1, pp. 611-628, 2008. 